\title{
REVISION OF BRACHYSTETHUS (HETEROPTERA, PENTATOMIDAE, EDESSINAE) ${ }^{1}$
}

\author{
Aline Barcellos ${ }^{2}$ \\ Jocélia Grazia ${ }^{3}$
}

\begin{abstract}
Brachystethus Laporte, 1832 is revised. Brachystethus coxalis Breddin, 1904, B. cribrus (Fabricius, 1781), B. geniculatus (Fabricius, 1787), B. improvisus Breddin, 1905, B. rubromaculatus Dallas, 1851, B. signoreti Stål, 1872, B. tricolor Bolívar, 1879, B. vexillum Breddin, 1903 and B. vicinus Signoret, 1851 are redescribed based on morphological characters, with emphasis on genitalia of both sexes. A new species, B. schuhi, from Guyana, is described, and B. discolor (Walker, 1867), incertae sedis, is removed from the genus. Illustrations and a key for species of Brachystethus are provided.
\end{abstract}

KEYWORDS. Edessinae, taxonomy, Neotropical, genitalia.

\section{INTRODUCTION}

Brachystethus was described by LAPORTE (1832) as a subgenus of Edessa; SPINOLA (1837) elevated Brachystethus to generic status. KIRKALDY (1909) transferred Brachystethus to Pentatomini, listing ten species: Brachystethus cribrus (Fabricius, 1781), B. geniculatus (Fabricius, 1787), B. rubromaculatus Dallas, 1851, B. vicinus Signoret, 1851, B. discolor (Walker, 1867), B. signoreti Stål, 1872, B. tricolor Bolívar, 1789, B. vexillum Breddin, 1903, B. coxalis Breddin, 1904 and B. improvisus Breddin, 1905. The genus was included by Rolston et al. (1980) in their key for Pentatomini genera of Western Hemisphere that have the abdominal tubercle aposed to metasternal carina.

A cladistic analysis of Brachystethus (BARCELLos \& GRAZIA, 2003) supported the monophyly of the genus and also of Edessinae. Brachystethus shares with Edessa Fabricius, 1803, Olbia Stål, 1862, Pantochlora Stål, 1870 and Peromatus Amyot \& Serville, 1843 the mesosternal carina not elevated, phallus with a short vesica and presence of a ventral, beak-like projection in the thickening of vaginal intima. Based on these characters, the reinclusion of Brachystethus in Edessinae was proposed. The monophyly of Brachystethus was also supported by synapomorphic characters: metasternal carina partially bifurcated, pygophore with blade-like processes, anterior margin of gonocoxites

1. Contribution number 405 of Departamento de Zoologia, Universidade Federal do Rio Grande do Sul.

2. Museu de Ciências Naturais, Fundação Zoobotânica do Rio Grande do Sul, Caixa Postal 1188, 90001-970, Porto Alegre, RS, Brazil. (alinebar@ig.com.br)

3. Departamento de Zoologia, Universidade Federal do Rio Grande do Sul, Av. Bento Gonçalves, 9500, Prédio 43435.2, 91501-970, Porto Alegre, RS, Brazil. (jocelia@ufrgs.br) 
9 medially concave and gonapophyses 9 with a medial thickening. The genus is herein revised and illustrated, with emphasis on morphology of external genitalia.

\section{MATERIAL AND METHODS}

A total of 390 specimens were examined, borrowed from the Institutions listed below. Except DARC, acronyms follow ARNETT et al. (1993); names of curators and/or responsible for loans are between parenthesis: AMNH, American Museum of Natural History, New York, USA (R.T. Schuh); BMNH, Natural History Museum, London, UK (J. Margerison-Knight); DARC, David A. Rider's Collection, North Dakota State University, Fargo, North Dakota, USA (D.A. Rider); DEIC, Institut fur Pflanzenschutzforschung der Akademie der Landwirtschaftswissenschaften, Eberswalde, Germany (A. Taeger); DZUP, Coleção de Entomologia Prof. Pe. Jesus Santiago Moure, Universidade Federal do Paraná, Curitiba, Brazil (L. Marinoni); INBC, Instituto Nacional de Biodiversidad, Heredia, Costa Rica (J.A.U. Gómez); IZAV, Instituto de Zoologia Agrícola, Universidad Central de Venezuela, Maracay, Venezuela (E. Osuna); MNCE, Museu de História Natural Capão da Embuia, Curitiba, Brazil (S. Malkowski); MNCS, Museo Nacional de Ciencias Naturales, Madrid, Spain (I. Izquierdo); MNHN, Museum National d'Histoire Naturelle, Paris, France (D. Pluot-Sigwalt); MNRJ, Museu Nacional, Universidade Federal do Rio de Janeiro, Rio de Janeiro, Brazil (M.A. Monné); MPEG, Museu Paraense Emílio Goeldi, Belém, Brazil (A. Harada); MRSN, Museo Regionale Scienze Naturali, Torino, Italy (P.L. Scaramozzino); MZSP, Museu de Zoologia, Universidade de São Paulo, São Paulo, Brazil (F. Du Val); NHMW, Naturhistorisches Museum, Viena, Austria (H. Zettel); SEAN, Museo Entomologico S.E.A., Leon, Nicaragua (J. Maes); UFRG, Departamento de Zoologia, Universidade Federal do Rio Grande do Sul, Porto Alegre, Brazil (J. Grazia); USNM, National Museum of Natural History, Washington D.C., USA (R.C. Froeschner); ZMUC, Zoological Museum, University of Copenhagen, Copenhagen, Denmark (N. Møller Andersen).

Type-specimens of Daimonocoris bolivianus Kormilev, 1951, Edessa marginatus Laporte, 1832, Edessa circumscripta Burmeister, 1835 and Brachystethus sexmaculatus Herrich-Schäffer, 1853 were not found.

A total of 16 morphometric parameters were measured; average, minimum and maximum values and standard deviation were calculated for ten males and ten females of each species. The measurements, in milimeters, were taken as in GRAZIA \& BARCELlos (1991), added with corium length, taken from the base of hemelitrum to posterolateral angle of corium. The genitalia were cleared with $10 \% \mathrm{KOH}$ and stained with Congo Red. Illustrations were made with drawing tube on stereomicroscope. The genitalia nomenclature followed Dupuis $(1955,1970)$ and Grazia \& Barcellos (1991). The females of $B$. coxalis (only one specimen available) and of B. tricolor (type-specimen) were not dissected. Data from geographical distribution were based on specimens labels; information from literature was not considered to avoid mistakes on species identification. The sequence of descriptions follows cladistic relationships among species, according to BARCELlos \& GraZIA (2003).

\section{Brachystethus Laporte, 1832}

Edessa (Brachystethus) LAPORTE, 1832:63; BURMEISTER, 1835:356.

Brachystethus; Spinola, 1837:343; Amyot \& Serville, 1843:160; Herrich-SchäFfer, 1845:1; 1853:310; Dallas, 1851:336; Signoret, 1851:344; Stål, 1867:532 (key); 1872:48; Walker, 1868:454; Distant, 1880:84; Berg, 1892:11; Lethierry \& Severin, 1893:187 (cat.); Bergroth, 1908:178; Kirkaldy, 1909:152 (cat.); Pirán, 1955:337; Rolston et al., 1980:122 (key); Froeschner, 1981:69 (cat.); Brailovsky, 1988:129 (diagnosis, distr.); Rider, 1991:584 (key).

Daimonocoris Kormilev, 1951:37; Pirán, 1955:337 (syn.).

Type species of Brachystethus, Cimex geniculatus Fabricius, 1787, by monotypy; of Daimonocoris, D. bolivianus Kormilev, 1951.

Diagnosis. Medium-sized, broad oval species (fig. 1), predominantly dull, but some species brightly colored (figs. 2-13). Head small, short, jugae contiguous before tylus; first antennal segment surpassing apex of head. Rostrum short, not surpassing mesocoxae. 


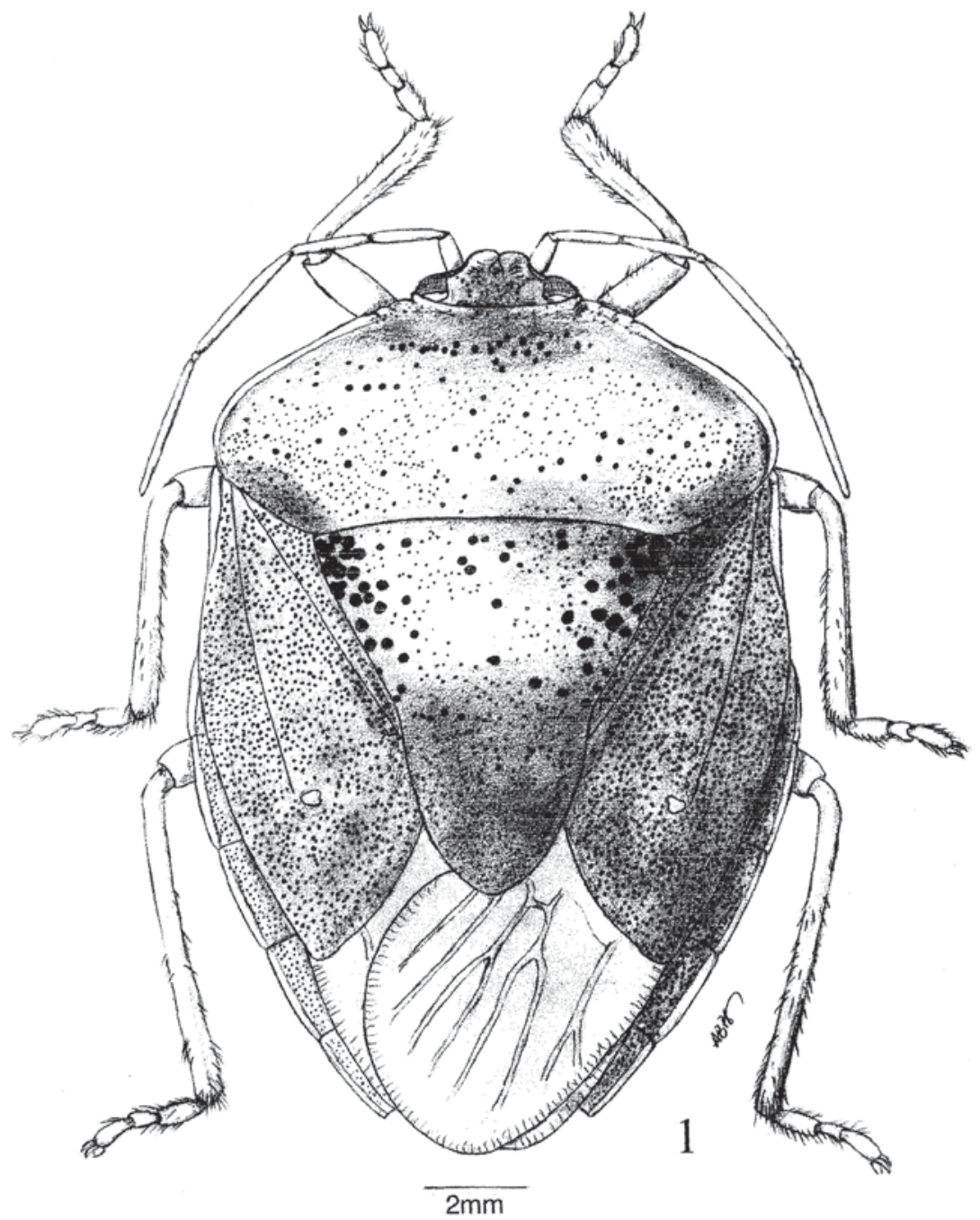

Fig. 1. Brachystethus geniculatus (Fabricius, 1787).

Metasternum elevated in an anteriorly partially bifurcated and posteriorly bifurcated carina, extended over part of mesosternum. Abdomen with medial tubercle apposed to the posterior margin of metasternal carina.

Description. Medium size (total length in $\mathrm{mm}, \sigma, 13-20.2, \varsigma, 14.9-22.4)$. Broad oval shape. Head about one and a half wider than long, with transversal wrinkles on jugae and tylus, and small sinuated wrinkles between oceli. Punctures concentrated in 1+1 narrow, semi-circular and divergent stripes that arise from base of oceli, directed forward until base of jugae, and in a median area between oceli, variable in length. Jugae rounded 
at apex and contiguous before tylus. Eyes large and prominent. Antennae with each segment progressively longer than the preceding. First antennal segment clearly surpassing apex of head. Bucculae elevated, anteriorly truncated, evanescent posteriorly. First rostral segment clearly surpassing them, by more than half of segment length. Rostrum not surpassing mesocoxae. Pronotum trapezoidal, strongly declivent on anterior half. Anterior margin slightly concave, openly U-shaped. Anterolateral angles prominent, forming a small tooth. Anterolateral margins slightly sinuous, subretilineous. Humeri rounded, little projected. Posterolateral and posterior margins straight. Punctures fine, more concentrated before cicatrices and regularly distributed over remaining disc of pronotum; among them, coarse punctures less frequent and irregularly distributed. Scutellum with broad and rounded apex, surpassing an imaginary line across middle of fifth connexival segment. Corium with punctures fine, dense and regularly distributed. Frenum slightly surpassing apical half of scutellum. Posterolateral angles rounded, attaining about the middle of sixth tergite. Membrane with more than five longitudinal and bifurcated veins. Tibiae dorsally sulcated. Ventral surface of thorax more densely punctured on propleura, mesoepisternum and metaepimerum. Evaporatory surface covering mesoepimerum and metaepisternum. Ostiolar rugae variable in length. Prosternum flat. Mesosternum slightly carinated. Metasternum carinated, produced over mesosternum. Anterior margin of metasternal carina with a deep median depression, partially bifurcated; posterior margin bifurcated, apposed to medial abdominal tubercle. Third urosternite with a stout medial tubercle. Posterolateral angles of connexivum slightly prominent. Spiracles elliptical. Trichobothria over or laterad to spiracular line.

Male genitalia. Pygophore ovoid to sub-quadrangular, shallowly open dorsally. Dorsal rim (dor) medially concave, forming or not $1+1$ lateral lobes (ldor) variable in size and shape among the species. Tenth segment $(\mathrm{X})$ trapezoidal, wider basaly than apically. Parameres (pa) uniramous. Genital cup with dorsally visible, blade-like processes (gcp). Posterolateral angles of pygophore rounded and tumid. Phallus: basal plate (bp) wide; phallotheca (ph) tubular, elongated, curved dorsad and strongly esclerotized; vesica (v) short, about $1 / 5$ of length of phallotheca, with $1+1$ dorsal, hook-like processes, convergent at apex, and only one ventral membranous lobe; ductus seminis distalis (dsd) short, not surpassing apex of vesica; conjunctiva absent.

Female genitalia. Laterotergites 8 and 9 (la8, la9) without apical spine. Spiracles on laterotergites 8 present. Laterotergites 9 not surpassing transverse band uniting laterotergites 8. Gonocoxites 9 ( $\mathrm{gc} 9$ ) broad, trapezoidal, anterolateral angles extended in arms variable in length and thickness; anterior margin medially concave. Gonapophyses 9 (g9) with a medial thickening (mt). Thickening of vaginal intima (tvi) with a ventral, beak-like projection, visible in profile, bearing the orificium receptaculi (or). Chitinellipsen (ch) present. Ductus receptaculi (dr) thin anteriorly to vesicular area, and variable after that. Capsula seminalis (cs) with three digitiform processes, directed toward annular crests (aac, pac).

Distribution. Mexico, Central and South America.

Comments. Brachystethus can be easily distinguished from other edessines by the broad, oval outline and the metasternal carina partially bifurcated. These characters, combined with the presence of blade-like processes on genital cup and of a medial thickening on gonapophyses 9, are diagnostic for the genus. The exam of the holotype of Ochlerus discolor, transferred to Brachystethus by KIRKALDY (1909), allowed to 
conclude that $B$. discolor should be removed from Brachystethus, since it presents morphological characters different from the remaining species of the genus, as the body outline, narrowly ovate, the first rostral segment concealed by bucculae and laterotergites 9 with acute apex, surpassing band joining laterotergites 8 dorsally. As the holotype presents one of the gonocoxites 8 deformed, and the metasternum can not be clearly observed because it is crossed by the pin, the species is here considered as incertae sedis.

\section{Key to the species of Brachystethus}

1. Metasternal carina, in profile, strongly elevated anteriorly ..............................................

Metasternal carina, in profile, flat or little elevated anteriorly .......................................... 7

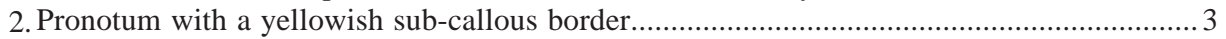

Pronotum without a yellowish sub-callous border........................................................... 5

3. Venter with longitudinal series of yellowish spots. Fourth antennal segment medially swollen..

B. cribrus (Fabricius)

Venter uniformly castaneous to dark-castaneous, lacking yellowish spots. Fourth antennal segment cylindrical.....

4. Lobes of dorsal rim of pygophore projected, well developed (fig. 22). Posterior borders of gonocoxites 8 sinuate, not projected over laterotergites 8 (fig. 63).

B. improvisus Breddin

Lobes of dorsal rim of pygophore scarcely projected (fig. 23). Posterior borders the gonocoxites 8 projected in acute angle, over laterotergites 8 (fig. 64). B. vicinus Signoret

5 . Hemelytra with a small yellowish callus, near and entad to apex of radial vein.

B. schuhi, sp. nov.

Hemelytra without callus

6. Apex of femora and base of tibiae yellowish. B. signoreti Stål

Legs uniformly castaneous to black.

B. rubromaculatus Dallas

7. Ostiolar rugae short, extending for $1 / 3$ the distance between inner margin ostiolum and lateral margin of metapleura. Metasternum carina with a medial, transversal sulcus.................... 8

Ostiolar rugae long, extending for 2/3 of distance between inner margin of ostiolum and lateral margin of metapleura. Metasternal carina lacking sulcus.

8. Dorsal surface black, with red spots on hemelytra, in variable extension (fig.8).

B. tricolor Bolívar

Dorsal surface black, with yellow spots on hemelytra, in variable extension (fig. 9).

B. vexillum Breddin

9. Coxae, trochanters, base and apex of femora, base of tibiae and tarsi yellowish.

B. coxalis Breddin

Legs uniformly castaneous to dark-castaneous B. geniculatus (Fabricius)

\section{Brachystethus rubromaculatus Dallas, 1851}

(Figs. 2, 3, 14, 24, 34, 44, 53-56, 65, 66)

Brachystethus rubromaculatus Dallas, 1851:337; Signoret, 1851:345; STÅL, 1862:118; MAYR, 1866:68; Walker, 1868:454; Stål, 1872:48; Distant, 1880:85, pl. 8, fig. 6; Lethierry \& Severin, 1893:187 (cat.); Kirkaldy, 1909:152 (cat.); Brailovsky \& BarRera, 1982:239 (redescription); Brailovsky, 1988:129, fig. 118 (diagnosis, distr.); Brallovsky et al., 1992:179, pl. 24, figs. 1-9 (nymphs, biology); Maes, 1994:8 (cat.).

Brachystethus sexmaculatus HeRrich-SchäFFER, 1853:310, fig. 1010; STÅL, 1862:118 (syn.). 


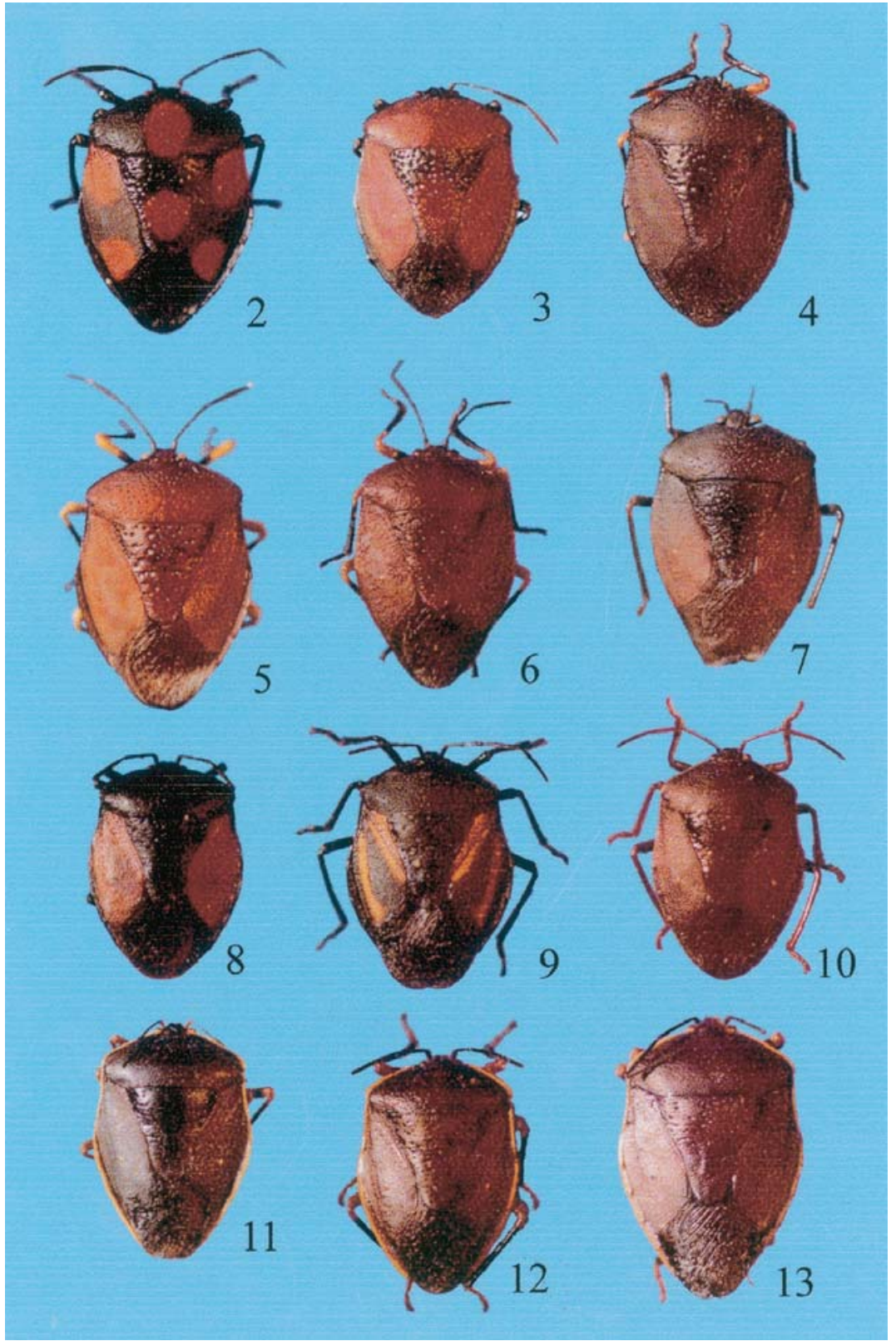

Figs. 2-13. 2, 3, Brachystethus rubromaculatus Dallas, 1851; 4, 5, B. signoreti Stål; 1872; 6, B. cribrus (Fabricius, 1781); 7, B. schuhi sp. nov.; 8, B. tricolor Bolívar, 1879; 9, B. vexillum Breddin, 1903; 10, B. geniculatus (Fabricius, 1787); 11, B. coxalis Breddin, 1904; 12, B. improvisus Breddin, 1905; 13, B. vicinus Signoret, 1851. Magnification 1.63X. 
Type material of $B$. rubromaculatus. Lectotype $\sigma$, here designated, labeled: a) Honduras/45123; b) "a"; c) Type; d) Dyson coll. (BMNH); paralectotype $\sigma$, here designated, labeled: a) Mexico; b) "b" (BMNH). Type locality: Honduras and Mexico.

Description. Dorsal surface black, with red spots of variable extension on pronotum, scutellum and corium (figs. 2, 3). Head entirely black, with conspicuous wrinkles. Punctures sparse; semi-circular stripes little conspicuous, moderately punctured. Antennae black; fourth antennal segment medially swollen, with longitudinal sulcus. Second antennal segment about one and a half as long as first; third slightly longer than second; fourth almost twice as long as third and slightly shorter than fifth. Pronotum black, with a large, rounded, red spot medially; in some specimens, extended laterally over most part of disc, except on a narrow black stripe on anterior and anterolateral margins. Punctures black, minute, regularly distributed on the disk of pronotum; among them, sparser and larger punctures. Scutellum black, with a rounded, red spot, which occupies the center of median-apical region; in some specimens, it extends anteriorly and posteriorly, covering the apical $2 / 3$ of scutellum. Surface of basal half sub-rugose, with large punctures regularly distributed. Apical half with fine punctures, more concentrated lateral and apically. Corium without callus, color pattern variable, usually black, with a large red spot on basal third and another on apical third, except along lateral margins. In some specimens, the corium is predominantly red, with black portion restricted to a thin lateral stripe and one small spot near to apex of radial vein. Ventral surface of thorax dark reddish-brown to black, with a red spot near to outer margin of metaepimerum; punctures fine, concolor. Ostiolar rugae extending by $2 / 3$ of distance between the inner margin of ostiolum and lateral margin of metapleura. Metasternum, in profile, strongly elevated anteriorly. Legs dark reddish-castaneous to black. Connexival segments entirely black; punctures fine, moderate and regularly distributed. Venter black to dark reddish-castaneous, with $1+1$ oblong red spots of variable length, generally extending from anterior margin of fourth sternite onto a little bit beyond the anterior margin of seventh. In some specimens, the spots can become coalescent, making the abdomen almost totally red, only with a lateral black stripe. Punctures fine, very sparse, if present restricted to medial area. Spiracles black.

Male genitalia. Pygophore trapezoidal, wider apically. Lobes of dorsal rim conspicuous, slightly projected posterad, rounded at apex. Genital cup processes moderately visible dorsally (figs. 14, 24). Parameres with wide apex, anterolaterally directed, a little wider than half length of tenth segment (fig. 44). Ventral rim broadly Vshaped (fig. 34). Phallus as described for the genus (figs. 53-55).

Measurements $(\mathrm{n}=10)$. Head length $2.08(1.92-2.44) \pm 0.15$, width $3.02(2.88-3.28) \pm$ 0.14 ; interocular distance $1.59(1.52-1.76) \pm 0.08$; anteocular length $0.68(0.6-0.8) \pm 0.07$; length of antennal segments: I - $0.81(0.72-0.88) \pm 0.06$; II - 1.24 (1.04-1.48) \pm 0.14 ; III - 1.45 $(1.28-1.68) \pm 0.11 ; \mathrm{IV}-2.79(2.4-3.28) \pm 0.24 ; \mathrm{V}-3.28(2.96-3.8) \pm 0.25$; pronotal length 4.36 (4.01-4.84) \pm 0.25 , width $9.74(8.85-10.69) \pm 0.53$; scutellar length $6.66(6.01-7.35) \pm 0.43$, width 6.16 (5.68-6.76) \pm 0.35 ; corium length 9.16 (8.52-10.02) \pm 0.45 ; abdominal width 10.99 (10.02-12.36) \pm 0.79 ; total length 14.69 (13.78-15.87) \pm 0.70 .

Female genitalia. Gonocoxites 8 with posterior borders convex and sutural borders parallel and contiguous, except at apex (fig. 56). Gonocoxites 9 more than three times wider across arms apices than long medially; anterior border broadly U-shaped. Arms of gonocoxites 9 with length equal to $1 / 4$ of plate width across apices of arms. Thickening of vaginal intima digitiform. Ductus receptaculi thin, about one and a half longer before than 

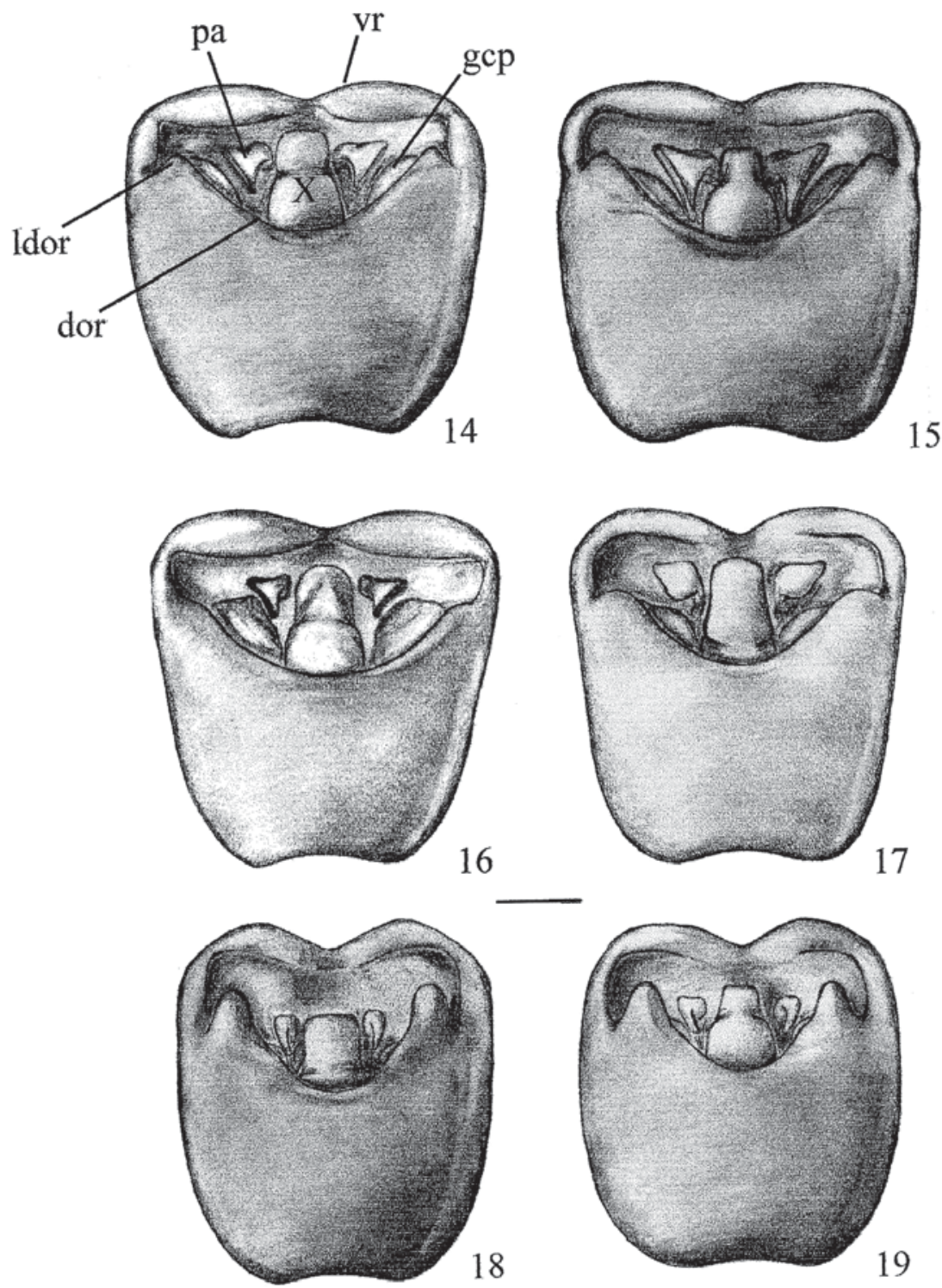

Figs. 14-19. Pygophore, dorsal: 14, Brachystethus rubromaculatus; 15, B. signoreti; 16, B. cribrus; 17, B. schuhi; 18, B. tricolor; 19, B. vexillum (dor, dorsal rim; gcp, genital cup process; ldor, lobe of dorsal rim; pa, paramere; vr, ventral rim; $X$, tenth segment). Scale, $1 \mathrm{~mm}$.

after vesicular area. Capsula seminalis globose. Annular crests convergent (figs. 65, 66).

Measurements $(\mathrm{n}=10)$. Head length $2.19(2-2.44) \pm 0.16$, width $3.25(3.08-3.44) \pm$ 0.14 ; interocular distance $1.71(1.56-1.84) \pm 0.09$; anteocular length $0.74(0.64-0.92) \pm 0.10$; 
length of antennal segments: I - $0.88(0.76-0.96) \pm 0.06$; II - $1.40(1.28-1.6) \pm 0.10$; III - 1.60 $(1.28-1.92) \pm 0.18 ; \mathrm{IV}-3.01(2.76-3.36) \pm 0.21 ; \mathrm{V}-3.41$ (3.28-3.56) $\pm 0.09 ;$ pronotal length $4.91(4.51-5.34) \pm 0.34$, width $10.97(10.10-11.77) \pm 0.63$; scutellar length $7.46(6.68-8.18) \pm$ 0.57 , width $7.02(6.35-7.60) \pm 0.47$; corium length 10.31 (9.35-11.36) \pm 0.68 ; abdominal width $12.46(11.27-13.69) \pm 0.88$; total length $16.15(15.03-17.37) \pm 0.90$.

Material examined. MEXICO, Veracruz: Atoyac (Paso del Macho, 5 mi. Southwest), $\subsetneq$, 24,25.VI.82, tropical forest 2500', Rolf L. Aalbu col. (DARC); Santiago (7 mi. North Tuxtla), o, 8.VII.63 (DARC); (Est. Biol. Los Tuxtlas), హ, 4.VII.1988, C. Mayorga (AMNH); హ, 22.VIII.1982, C. O'Brien, L. O'Brien \& Wibmer, 250' (DARC); Las Cabañas, f, 28.V.1953, L. Vazquez (AMNH); Fortin, o', 23.VII. 1989 (DARC); La Buena Ventura, ક, 14.VII.09, M.Y.B. (AMNH); Catemaco (5 mi. East), J', \&, 2-4.VII.1971, Murray, Hart \& Schaffner (DARC); Lake Catemaco, $\subsetneq$, 23.VII.1955, P. \& C. Vaurie, 1000' (AMNH); З, 16.VI.1969, Bright \& Campbell (DARC); Yucatán: Chichén-Itzá, ‡, 10.VIII.1990, D.A. Rider (DARC); Oaxaca: Matias Romero (17 mi. North), ơ, 27.VI.1971, C.W. O'Brien (DARC); Tehuantepec: Isth. of Tehuantepec, $f$, Sumichrast, Collection Ashmead (USNM); Cinta Lapa Valley, f, V.44, E. Hernandez (USNM); Quintana Roo: Klan Nuevo, f, 6, 7.VI.1959, P. \& C. Vaurie (AMNH); Tabasco: Villahermosa (5 mi. South), $\&$, 13.VI.65, Burke, Meyer \& Schaffner (DARC); Chiapas: Ruins at Palenque, f, 26-30.VI.1959, P. \& C. Vaurie (AMNH); Huatimac, $\checkmark$, V.1958, Patiño col. (MZSP). GUATEMALA, Garcia (Dona Maria Zacapa), \&, 20.IX.81 (DARC); Retalhuleu: S. Sebastian, $25^{\circ}, \sigma$, L. Thiel (USNM); Alta Verapaz: Chacoj, $\subsetneq$, Champion col., ex Godman \& Salvin (BMNH); Verapaz, Teleman, o', Champion, ex. Godman \& Salvin (AMNH). HONDURAS, Yoro: Subirana, $\mathscr{f}$, II, Stadelmann (AMNH). NICARAGUA, Zelaya: El Recreo, $\mathscr{f}$, X.1984, J.M. Maes (SEAN). COSTA RICA, $\subsetneq$, (AMNH); Guanacaste: La Cruz (6 mi. North), $\subsetneq$, 19.VI.1974, C.W. O’Brien, L. O'Brien \& Marshall (DARC); Alajuela: Zapote de Upala (vic. Bijagua), f, 8.XII.1972, R. Ortiz (DARC); Heredia: Puerto Viejo, f, 8.VIII.1965, G.W. Frankie (DARC); (2 Km South, La Sielva Biol. Sta.), 2 3, 2 \&, 3-5.VI.1984, Riley, Rider \& Le Doux (DARC); Cartago: Iica (Turrialba), §, VIII.1963, C.C. Porter (AMNH); Port Parker (Elena Bay), f, 13.I.1938, Zcca Exped. Acc. 37483 (AMNH); Puntarenas: Santa Elena (6 Km South),, , 6, 7.VI. 1983, J.E. Wappes (DARC); Ricon de Osa (Osa Peninsula), 7 3, 2 \& 14-26.VII.1969, Toby Schuh \& Janet Crane (AMNH); San José: §, C.P. Dodge (AMNH). PANAMA, Chiriquí: David, ろ, Champion (AMNH); 3, ex Godman \& Salvin (AMNH); V. de Chiriquí, f, ex Godman \& Salvin, 25-4000ft (AMNH); Panama: Summit, $\mathcal{\sigma}$, XII.1953, N.L.H. Krauss (AMNH); Madden Forest, $\mathfrak{f}, 18$. V.1980, Riley \& Le Doux (DARC); F.T. Kobbe, $\subsetneq$, 28.VI.1974, C.W. O’Brien, L. O’Brien \& Marshall, at night (DARC). No data: §, F 1672 (AMNH); , Brachystethus rubromaculatus det. Dallas, H.G.B., F 1673 (AMNH); f, F 1672; , 1955 (MNHN); † (MZSP).

Distribution. Mexico (Veracruz, Yucatán, Oaxaca, Tehuantepec, Quintana Roo, Tabasco, Chiapas), Guatemala (Retalhuleu, Alta Verapaz), Honduras, Nicaragua, Costa Rica (Guanacaste, Alajuela, Heredia, Cartago, Puntarenas, San José), Panama (Chiriquí, Panama).

Comments. Brachystethus rubromaculatus is the sister group of the clade formed by $B$. signoreti, B. cribrus and B. schuhi, sharing with these species the fourth antennal segment medially swollen, the metasternal carina strongly elevated anteriorly and the little projected lobes of dorsal rim of pygophore (BARCELLOS \& GRAZIA, 2003). It can be easily distinguished by the most frequent color pattern, dorsally black, with rounded red spots and venter black, with $1+1$ spots extending from the anterior margin of fourth sternite until anterior margin of seventh. However, in specimens in which the red spots are coalescent, $B$. rubromaculatus can be confounded with $B$. signoreti, but easily distinguished by the color pattern of legs, uniformly black, the finer punctuation of scutellum and by genitalia, especially the size of parameres in relation to $\mathrm{X}$ segment. Legs uniformly black also distinguish B. rubromaculatus from B. cribrus and B. schuhi. 

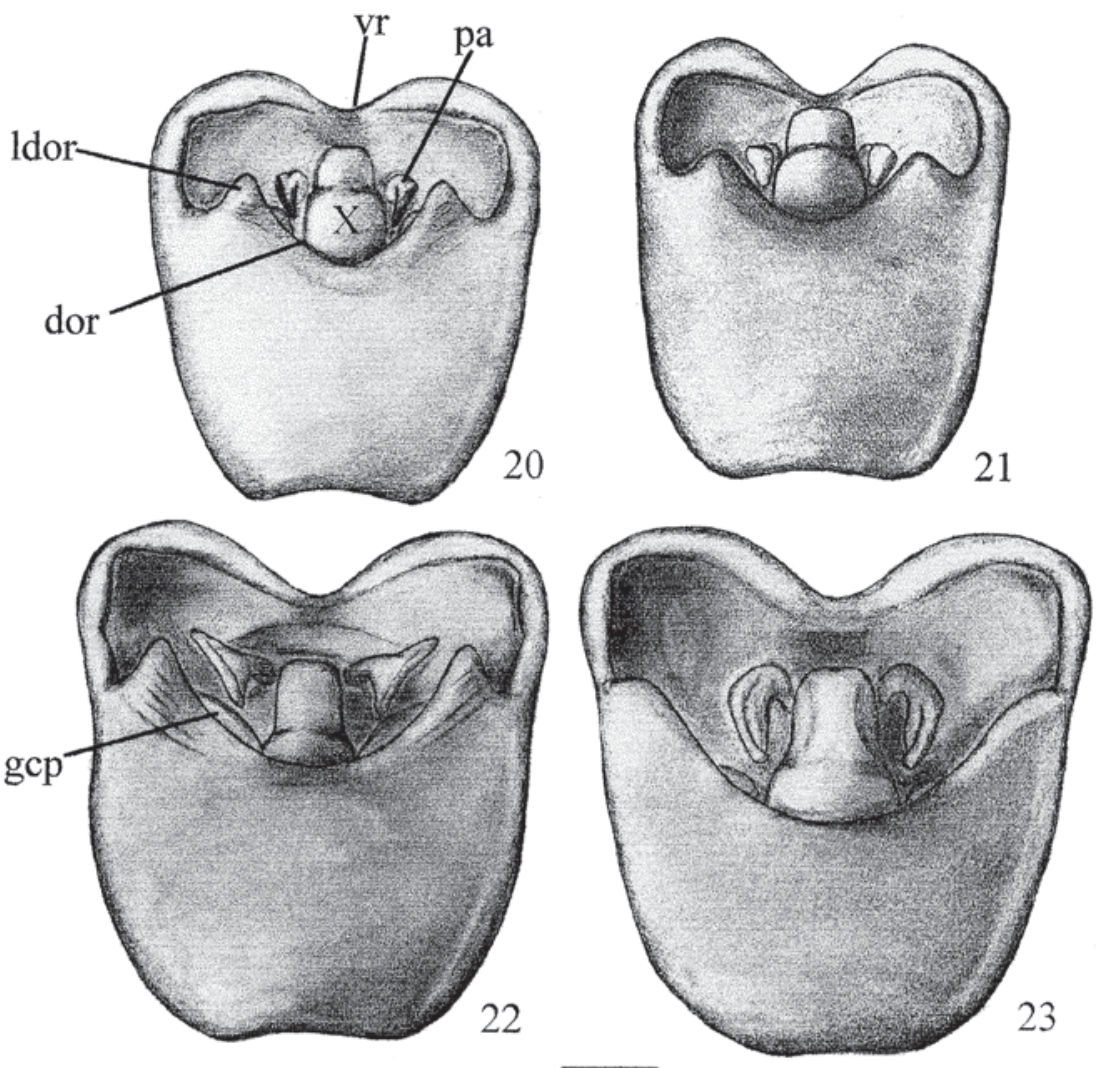

Figs. 20-23. Pygophore, dorsal: 20, Brachystethus geniculatus; 21, B. coxalis; 22, B. improvisus; 23, B. vicinus (dor, dorsal rim; gcp, genital cup process; ldor, lobe of dorsal rim; pa, paramere; vr, ventral rim; $X$, tenth segment). Scale, $1 \mathrm{~mm}$.

Brachystethus signoreti Stål, 1872

(Figs. 4, 5, 15, 25, 35, 45, 57, 67)

Brachystethus geniculatus Signoret, 1851:345 (preoccupied, not Fabricius, 1787); WalKer, 1868:455. Brachystethus signoretii STÅL, 1872:48 (new name); KIRKALDY, 1909:152 (cat.); GraZIA, 1984:74. Brachystethus signoreti; LeTHIERRY \& SEVERIN, 1893:187 (cat.).

Type material. Lectotype $\sigma$, here designated, labeled: a) Laguaira, Coll. Signoret; $b$ ) geniculat. det. Signoret (NHMW); paralectotype of, here designated, labeled: a) Laguaira, Coll. Signoret; b) geniculata det. Signoret (NHMW). Type locality, Venezuela ("La Guaira").

Description. Dorsal surface dark- to reddish-castaneous (figs. 4, 5). Head dark- to reddish-castaneous, with conspicuous wrinkles. Punctures of median stripe sparse; semicircular stripes moderately punctured. Antennae black; fourth antennal segment medially swollen, with a longitudinal sulcus. Second antennal segment about one and a half as long as first, third about one and a quarter the second, fourth almost twice the 
third and fifth slightly longer than fourth. Pronotum dark- to reddish-castaneous. Punctures fine regularly distributed, interposed with coarse punctures, more concentrated behind cicatrices; medium-sized punctures densely distributed before cicatrices. Scutellum concolor with pronotum; coarse punctures distributed on basal 3/4, among them, fine punctures, denser laterally. Corium without callus, dark- to reddish-castaneous; on the reddish-castaneous specimens, present two darker, grayish stripes, along outer margin and entad to radial vein. Ventral surface of thorax with black, medium-sized punctures. Ostiolar rugae extending by $2 / 3$ of distance between inner margin of ostiolum and lateral margin of metapleura. Metasternum, in profile, strongly elevated anteriorly. Legs dark-castaneous to black, yellowish at apex of femora and base of tibiae. Connexival segments dark- to reddish-castaneous. Venter dark- to reddish-castaneous, in this case with reddish areas around and entad to spiracles and little less conspicuous sub-medially. Punctures fine, concolor, regularly distributed, but rare on medial fifth of abdomen. Spiracles black.

Male genitalia. Pygophore trapezoidal, wider apically. Lobes of dorsal rim conspicuous, little projected posterad, rounded at apex. Genital cup processes moderately visible dorsally (figs. 15, 25). Parameres very wide apically; apex antero-laterad directed, slightly longer than medial length of tenth segment (fig. 45). Ventral rim openly U-shaped (fig. 35).

Measurements $(n=10)$. Head length $1.93(1.84-2.04) \pm 0.06$, width $2.95(2.88-3.12) \pm$ 0.09 ; interocular distance $1.55(1.44-1.6) \pm 0.06$; anteocular length $0.66(0.60-0.72) \pm 0.05$; length of antennal segments: I - $0.82(0.80-0.88) \pm 0.03$; II - $1.22(1.08-1.4) \pm 0.08$; III - 1.51 $(1.24-1.68) \pm 0.15 ; \mathrm{IV}-2.93(2.72-3.2) \pm 0.17 ; \mathrm{V}-3.35(3.28-3.44) \pm 0.07$; pronotal length 4.23 (4.01-4.51) \pm 0.18 , width $9.44(8.85-10.10) \pm 0.39$; scutellar length $6.42(6.01-6.93) \pm 0.31$, width 6.01 (5.68-6.35) \pm 0.22 ; corium length $9.19(8.85-9.69) \pm 0.30$; abdominal width 10.55 (10.02-11.02) \pm 0.35 ; total length $14.35(13.78-15.03) \pm 0.46$.

Female genitalia. Posterior borders of gonocoxites 8 convex over laterotergites 9 . Sutural borders of gonocoxites 8 parallel and contiguous, except at apex (fig. 57). Gonocoxites 9 almost three and a half times wider across arms apices than long medially; anterior border broadly U-shaped. Length of arms equal to 1/6 of plate width. Thickening of vaginal intima digitiform. Ductus receptaculi thick after vesicular area, and about one and a half longer before than after this area. Capsula seminalis elongated, digitiform. Annular crests parallel (fig. 67).

Measurements $(n=10)$. Head length $2.09(1.92-2.28) \pm 0.12$, width $3.11(3.04-3.20) \pm$ 0.06 ; interocular distance 1.61 (1.52-1.68) \pm 0.06 ; anteocular length $0.70(0.60-0.80) \pm 0.06$; length of antennal segments: I - $0.94(0.84-1.12) \pm 0.08$; II - $1.40(1.28-1.52) \pm 0.08$; III - 1.68 $(1.36-1.84) \pm 0.15 ; \mathrm{IV}-3.18(2.76-3.36) \pm 0.18 ; \mathrm{V}-3.62(3.56-3.68) \pm 0.08$; pronotal length $4.72(4.51-5.01) \pm 0.14$, width $10.60(10.19-11.02) \pm 0.25$; scutellar length $7.18(6.93-7.35) \pm$ 0.13 , width $6.75(6.51-7.01) \pm 0.20$; corium length $10.27(9.94-10.69) \pm 0.22$; abdominal width $11.87(11.44-12.36) \pm 0.28$; total length $16.30(15.53-17.20) \pm 0.56$.

Material examined. VENEZUELA, $\subsetneq$, pres. by Perth Museum (BMNH 1953-629); $\subsetneq$, Schauf. col., Coll. Mayr (NHMW); San Julian, 2 ơ, VII.1900, W. Robinson Bequest (USNM 1929); Rancho Grande, 3 o', 2 \&, 19,26,27.IV.1945 (AMNH); Aragua: Villa de Cura (Est. Exp. Cataurito, $( \pm 1000 \mathrm{~m})$, f, 27.X.81, J.L. Garcia (IZAV); Maracay (450m, en luz), 2 \&, 22.V.1963, E. Osuna (AMNH, IZAV); Mérida: Mérida, 4 ơ, 3 (AMNH); Tachira: San Cristobal (River Black), \&, 7.III.1980, Miguel G. (IZAV); †, 5.I.1982, Gaban (IZAV); (Colon), †, 1.IV.1982, Gaban (IZAV); ơ, 10.X.1981, Ramirez (IZAV). ECUADOR, Pichincha: Santo Domingo de los Colorados (amazonian rainforest), ১, \&, X.1981, G. Onore (BMNH 1982-246); La Union, 2 of, f, 12.VII.1984, R. Haswell (DARC); Cotopaxi: 
(1500 m, tropical rainforest general collecting), $\subsetneq$, X.1982, G. Onore, (BMNH, 1982-246); Paramba, ‡, "Coll. I.R. Sc. N.B." (DARC); 4 †, Rosemberg col. (USNM); (3500', dry season), \&, II.97, Rosenberg (BMNH); Balrahamba, ơ, F. Campos (USNM); హ, 18.V.1935, F. Campos; J.C. Lutz Collection (USNM 1961); Pastaza: Canelos, ơ, Schouteden (DARC); Tungurahua: Ambato, ơ, 2 , Coll. Noualhier $1898(\mathrm{MNHN})$; , E. de Ville \& M.R.Belg col., Coll. Noualhier 1898 (MNHN). No data: $\subsetneq(\mathrm{BMNH})$; §, Coll. Mayr (NHMW).

Distribution. Venezuela (Aragua, Mérida, Tachira), Ecuador (Pichincha, Cotopaxi, Pastaza, Tungurahua).

Comments. Brachystethus signoreti is the sister group of the clade composed by B. cribrus and B. schuhi, sharing the homoplastic character of color pattern of legs, apex of femora and base of tibiae yellowish (BARCELLOS \& GRAZIA, 2003). It can be distinguished from these species by the absence of calli on the corium and from B. cribrus, especially, by the color of venter, lacking yellow spots. Brachystethus signoreti is also close to $B$. rubromaculatus, distinguished by the color pattern of legs, the coarser punctures of scutellum and, on male genitalia, especially by the larger parameres.

\section{Brachystethus cribrus (Fabricius, 1781)}

(Figs. 6, 16, 26, 36, 46, 58, 68)

Cimex cribrum Fabricius, 1781:357; 1794:117; Gmelin, 1793:2152.

Edessa cribrum; FABricius, 1803:154; BuRMEISTER, 1835:356.

Brachystethus cribrum; Aмyot \& Serville, 1843:161; Herrich-SchäFFer, 1845:1, fig. 788; Dallas, 1851:337; Herrich-SchäFfer, 1853:310; Signoret, 1851:344; Walker, 1868:455; StÅL, 1872:48;

Lethierry \& Severin, 1893:187 (cat.); Kirkaldy, 1909:152 (cat.); Pirán, 1956:31.

Brachystethus cribrus; Froeschner, 1981:69 (cat.).

Type material. Holotype o, labeled: Cimex cribrum F., det. P.H. V. Doesburg (ZMUC). The holotype of $C$. cribrum had been considered lost by the curator of ZMUC. ZIMSEN (1964) lists three syntypes of Cimex geniculatus and none of $C$. cribrum. The exam of one of the supposed syntypes of $C$. geniculatus showed that it had been identified as $C$. cribrum by Peter Von Doesburg, from Leiden Museum. In fact, this specimen presents the apex of femora and the base of tibiae yellowish, as well as yellowish spots on abdomen, fitting the Fabricius description of $C$. cribrum ("abdominis punctis genubusque flavis..."), that may indicate a mistake on the organization of Fabricius types, previously to ZIMSEN (1964) catalog. Thus, this specimen is here considered the holotype of Brachystethus cribrus. Type locality: Suriname.

Description. Dorsal surface dark reddish-castaneous, with a thin marginal yellowish stripe (fig. 6). Head dark-castaneous, sometimes with yellow margin; wrinkles conspicuous. Punctures of median stripe sparse, more frequent on $1+1$ semi-circular stripes and jugae. Antennae dark-castaneous to black; fourth antennal segment medially swollen, with longitudinal sulcus. Second antennal segment one and a half to almost twice as long as first; second and third subequal, fourth more than twice as long as third; fifth the longest, slightly longer than fourth. Pronotum dark reddish-castaneous, with yellowish stripe on anterolateral margins. Surface rugose; coarse punctures behind cicatrices, sparse over the disc. Punctures fine among the coarser ones, concentrated before cicatrices. Scutellum reddish-castaneous with coarse punctures more frequent on basal 2/3. Corium reddish-castaneous with yellowish callus lateral and entad the apex of radial vein. Ventral surface of thorax dark reddish-castaneous with medium-sized punctures. Ostiolar rugae extending by $2 / 3$ of distance between inner margin of ostiolum and lateral margin of metapleura. Metasternum, in profile, strongly elevated anteriorly. 

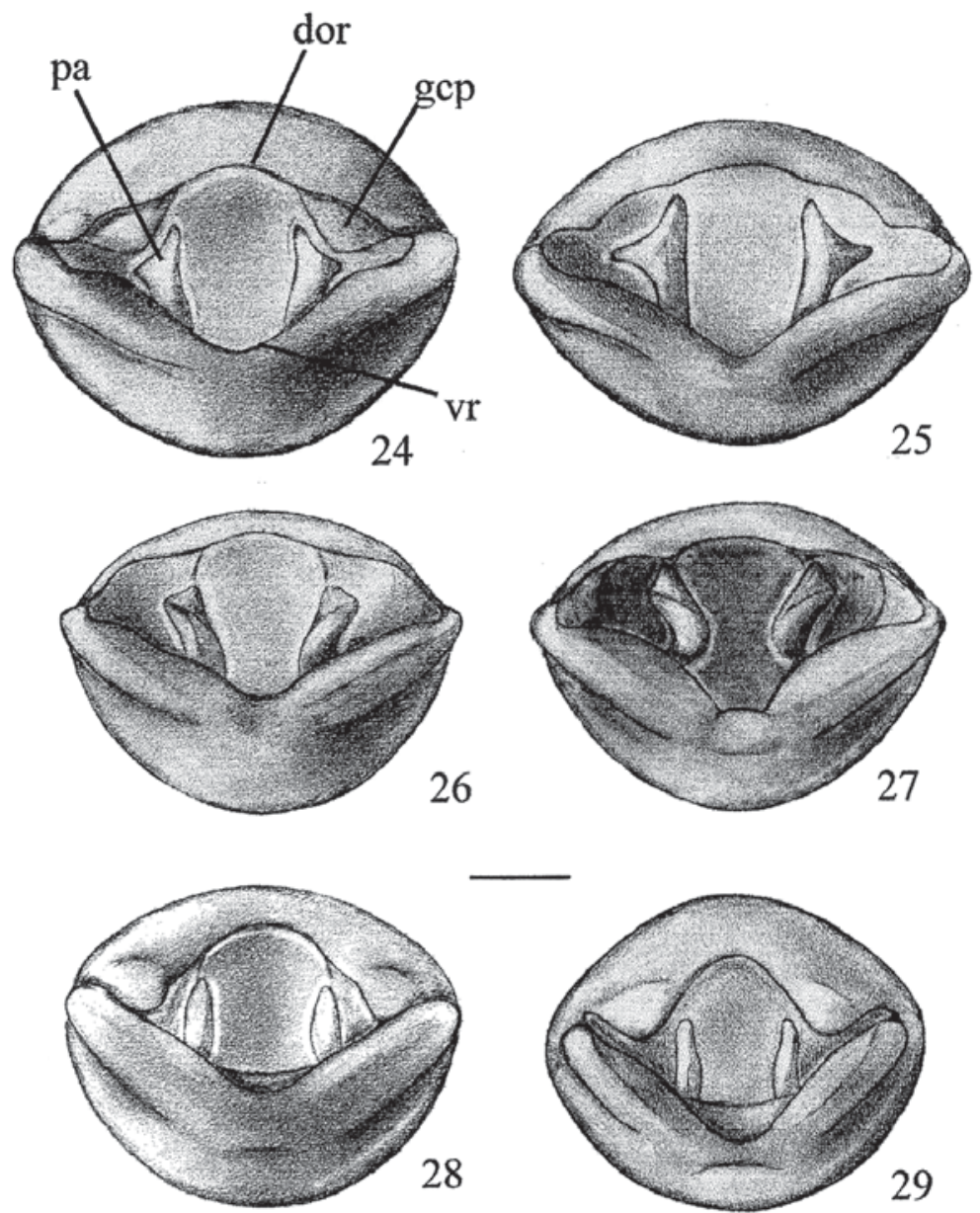

Figs. 24-29. Pygophore, caudal (tenth segment omitted): 24, Brachystethus rubromaculatus; 25, B. signoreti; 26, B. cribrus; 27, B. schuhi; 28, B. tricolor; 29, B. vexillum (dor, dorsal rim; gcp, genital cup process; pa, paramere; vr, ventral rim). Scale, $1 \mathrm{~mm}$.

Legs dark-castaneous, yellowish at apex of femora and base of tibiae. Connexival segments dark- to reddish-castaneous, narrowly bordered with yellow or light reddishcastaneous, over marginal sixth of each segment. Punctures fine, more concentrated entad to this stripe. Venter dark-castaneous, lateral margins with yellowish subcaloused stripe. Entad to spiracles, two longitudinal series of rounded yellow spots, one per segment, disposed in two rows: one lateral, on sternites III to VII and one median on sternites III to VI. This pattern varies intraspecifically; in some specimens, the external line presents two spots per segment on sternites III to VI. In some darker specimens, the spots may become less visible. Punctures fine, more concentrated along posterior margin of sternites and on lateral thirds of venter. Spiracles yellowish-castaneous to black. 
Male genitalia. Pygophore trapezoidal, wider apically. Lobes of dorsal rim inconspicuous. Genital cup processes broadly visible dorsally (figs. 16, 26). Parameres distally wide (fig. 46). Ventral rim broadly V-shaped (fig. 36).

Measurements $(n=10)$. Head length $1.90(1.76-2.04) \pm 0.09$, width $2.98(2.88-3.08) \pm$ 0.07 ; interocular distance $1.50(1.44-1.60) \pm 0.07$; anteocular length $0.65(0.56-0.72) \pm 0.05$; length of antennal segments: I - $0.90(0.80-0.96) \pm 0.05$; II - 1.32 (1.04-1.52) \pm 0.13 ; III - 1.44 (1.36-1.60) $\pm 0.09 ;$ IV - 3.07 (2.64-3.68) $\pm 0.32 ; \mathrm{V}-3.42$ (2.96-4.08) \pm 0.33 ; pronotal length 3.97 (3.67-4.26) \pm 0.18 , width 9.53 (9.19-10.02) \pm 0.27 ; scutellar length 6.45 (6.18-6.68) \pm 0.20 , width $6.04(5.68-6.51) \pm 0.22$; corium length $8.98(8.52-9.35) \pm 0.32$; abdominal width $10.30(9.77-11.02) \pm 0.39$; total length $14.14(13.53-14.78) \pm 0.44$.

Female genitalia. Posterior borders of gonocoxites 8 straight over laterotergites 9. Sutural borders of gonocoxites 8 parallel (fig. 58). Gonocoxites 9 about three times wider across arms apices than long medially; anterior border broadly U-shaped. Arms of gonocoxites 9 short, length equal to $1 / 6$ of plate width. Thickening of vaginal intima ogival. Ductus receptaculi thin after vesicular area; slightly more than one and a half longer before than after this area. Capsula seminalis elliptical. Annular crests convergent (fig. 68).

Measurements $(n=10)$. Head length $2.11(2.00-2.40) \pm 0.12$, width $3.20(3.04-3.36) \pm$ 0.09 ; interocular distance 1.66 (1.60-1.76) \pm 0.07 ; anteocular length $0.68(0.56-0.80) \pm 0.07$; length of antennal segments: I - $0.85(0.72-1.12) \pm 0.11$; II - $1.49(1.28-1.68) \pm 0.14$; III - 1.60 $(1.48-1.76) \pm 0.09 ; \mathrm{IV}-3.39(2.88-3.64) \pm 0.25 ; \mathrm{V}-3.67$ (3.20-3.92) \pm 0.25 ; pronotal length $4.79(4.18-6.18) \pm 0.58$, width $10.69(9.85-11.61) \pm 0.51$; scutellar length 7.37 (6.68-7.85) \pm 0.35 , width $6.81(6.26-7.35) \pm 0.31$; corium length 10.14 (9.27-10.69) \pm 0.57 ; abdominal width $11.92(11.02-12.86) \pm 0.62$; total length $16.22(14.86-17.62) \pm 0.83$.

Material examined. GUYANA, o, f, 1943, Bartlett col. (BMNH 1943-60, 1009); ठ, f, 20.II.1937, R. Pomaron \& A.S. Pinkus (AMNH); Kartabo (Distrito Bartica), $\subsetneq$, 1922 (BMNH 1954631); 3 3, 4 f, 13.VI, 16, 30.VII, 11.VIII.1922, M.D. Haviland, d.d. Collegium Newnhamense (BMNH 1954-631). SURINAME, Brokopondo: Brownsberg, 3 ơ, 3 \&, 19-21.VIII.1975, L.H. Rolston (DARC); Brownsberg Nat. Pk., ơ, 19-22.VII.1975, D. Engleman (AMNH). VENEZUELA, Amazonas: Santa Lucía, 3, 15-21.XI.1982, A. Chacon \& G.Yepes (IZAV). BRAZIL, Amazonas: Tefé, f, Raulin, Thayer Expedition (AMNH); Benjamin Constant (Rio Javari), ơ, 3 f, 1.II-15.III.1942, August Rabaut (AMNH); Rio Caiary-Uaupes, §, 1906, H. Schmidt (AMNH); Rio Negro (Tupurumara), ₹, 9.II.1963, J. \& B. Bechyné (MPEG); Pará: Peixe-Boi, f, 14.IV.1977, W. L. Overal. (MPEG); ठ̊, ‡, P. Waldir (MPEG); Jacareacanga, $\subsetneq$, X.69, R. Barbosa (AMNH); Itaituba (Santarensinho, Rio Tapajós), 2 ङ, f, V.1962, Dirings (MZSP); Santa Maria do Guamá, ơ, 2 f, 26.II.1987, P. Tadeu, J. Dias (MPEG); 3, 26.II.1987 (MPEG); Belém (Mocambo), 2 \&, 21.XII.1978, A. Ramos (MPEG); Óbidos (bx/ Amazonas), उ, Dirings (MZSP); Bujaru, 3, 20.IV.1979, R.B. Neto (MPEG); Maranhão: Alto Turiaçu (Aldeia Gurupluna), ơ, 19-22.II.1990, Helder Queiroz (MPEG); Mato Grosso: $10^{\circ} 25^{\prime} \mathrm{S} 59^{\circ} 28^{\prime} \mathrm{W}$ (300m), f, 17-22.III.1977, D. Engleman (AMNH); Rondônia: Ariquemes (62 Km SW, Fazenda Rancho Grande), ठ', 6-15.XII.1990, D.A. Rider \& J.E. Eger (DARC); (Linea C-20, 7 Km E. B-65), \&, 11-22.XI.1991, T.J. Riley (DARC); Ouro Preto do Oeste (Reserva INPA), ठ゙, 26.III.1985, F.F. Ramos (MPEG); Vilhena, \&, 17.XII.1986, C. Elias (DZUP); Pernambuco: Cabo, హ, Distant Collection (BMNH 1911-383); Goiás: Goiânia, f, I.1936, Borgmeier \& S. Lopes (UFRG). ECUADOR, Pichincha: Puerto Quito, ठ, V.1982, G. Onore (BMNH 1982-246); Napo: Coca, \&, ‡, XI,XII.1982, G. Onore (BMNH 1983-255); (tropical rainforest general collecting), 3 of, \&, XI.1982, G. Onore (BMNH 1982-246); (Pompeya on Napo R., $40 \mathrm{~km}$ from Coca), ö, V.1965, L.E. Pena (AMNH); Loja: S.E. Loja (Rio Jumboe, tributary Zamora R.), f, 1-IV-1965, idem (AMNH); Zamora Chinchipe: Zamora (35 Mi Loja), $\precsim$,, , 1-5.IV.1965, idem (AMNH). PERU, Ucayali: Pucallpa (Alt. 600 ft.), $\sigma$, 11.XI.1946, J.C. Pallister (AMNH); La Libertad: Cumpang (above Uctubamba, 2625m), $\sigma$, 13.X.1979, L. J. Barkley (DARC); Huánuco: 2 f, 23.XI.1946, Alt. 2200 ft., J.C. Pallister col. (AMNH); Tingo María (Alt. 2200 ft.), ơ, 2 \&, 23.IX, 21.X.1946, J.C. Pallister col. (AMNH); Cuzco: 

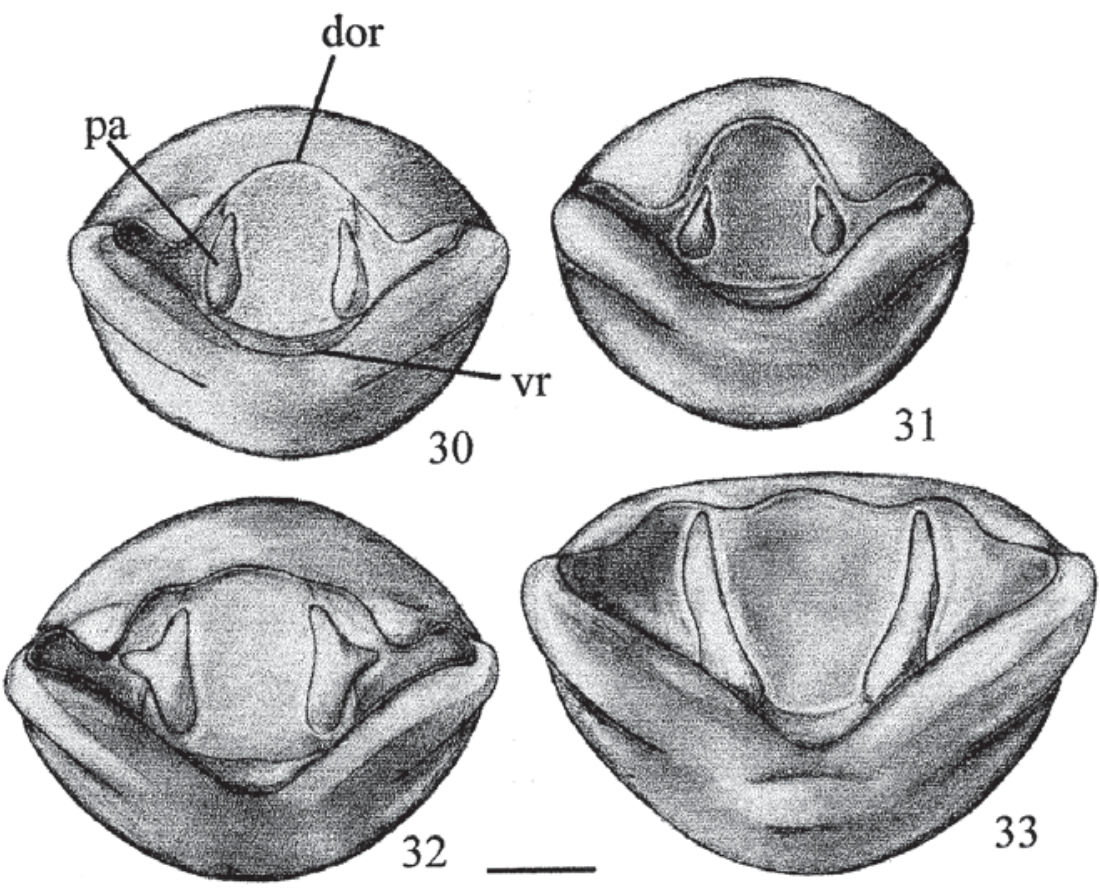

Figs. 30-33. Pygophore, caudal (tenth segment omitted): 30, Brachystethus geniculatus; 31 , B. coxalis; 32, B. improvisus; 33, B. vicinus (dor, dorsal rim; pa, paramere; vr, ventral rim). Scale, $1 \mathrm{~mm}$.

Cosñipata, \&, 6, 7.X.1966, Carrasco (UFRG); Pillcopata, 3, 11.X.68 (UFRG); Junín: Jauja Prov., Satipo, \&, V.1945, P. Paprzycki (AMNH); Rio Santiago, f, 23.XI.1924, F. 6012, H. Bassler Collection Acc. 33591 (AMNH); Rio Morona, f, 17.I.1925, F. 6194, H. Bassler Collection Acc. 33591 (AMNH); Upper Rio Maranon, 2 ơ, 26.VIII, 11.X.1924, F.6177, 6162, H. Bassler Collection Acc. 33591 (AMNH); Middle Rio Maranon, ơ, 8.XI.1925, H. Bassler Collection Acc. 33591 (AMNH); Tarapoto Region, ‡, 6.I.1926, F. 6042, H. Bassler Collection, Acc. 33591 (AMNH); Achinamiza, ö, 3 \&, 14.X, 20.XI.1927, F. 6001, H. Bassler Collection Acc. 33591 (AMNH); Moyobamba Region, ơ, 2 \&, 12.I.1925, 8.I.1926, 5.XII.1945, F. 6097, F. 6149, F. 6072, H. Bassler Collection Acc. 33591 (AMNH). BOLIVIA, 3 đ, 2 f, 1904, J. Steinbach col. (BMNH 1904-311);, , 1898, Coll. Noualhier (MNHN); Santa Cruz: Buena Vista Prov., Tohito, 400m, 2 f, 1956, Franz Steinbach (AMNH); Cochabamba: Rio Cristal Mavu (50 mi NE Cochabamba), 4 o, 4 \&, 30.IX, 27.X, 12, 30.XI.1949, L. Pena (AMNH, DARC). No data: $\sigma$,, , (AMNH); San Jose, $\zeta$ (MRSN).

Distribution. Guyana, Suriname (Brokopondo), Venezuela (Amazonas), Brazil (Amazonas, Pará, Maranhão, Mato Grosso, Rondônia, Pernambuco, Goiás), Ecuador (Pichincha, Napo, Loja, Zamora Chinchipe), Peru (Ucayali, La Libertad, Huánuco, Cuzco, Junín), Bolivia (Santa Cruz, Cochabamba).

Comments. Brachystethus cribrus is the sister species of B. schuhi, sharing a homoplastic character, presence of yellowish calli on corium (BARCELLOS \& GRAZIA, 2003). Brachystethus cribrus is separated from all other congeners by autapomorphic characters: abdomen with yellow spots and genital cup processes widely visible dorsally. It can also be distinguished from $B$. schuhi by homoplastic characters - pronotum with yellowish 
border, yellowish coxae and the inconspicuous lobes of dorsal rim. The yellowish margin of pronotum and the color pattern of coxae, femora and tibiae of $B$. cribrus are similar to those of B. coxalis, B. improvisus and B. vicinus, but can be easily distinguished by the yellow spots on the ventral surface of abdomen, the fourth antennal segment medially swollen and, on male genitalia, by the genital cup processes widely visible dorsally.

\section{Brachystethus schuhi sp. nov.}

(Figs. 7, 17, 27, 37, 47)

Etymology. Named in honor to Dr. Randall T. Schuh, for his great contribution to the knowledge of Heteroptera.

Type material. Holotype ơ, Guyana, no locality, no date, Babcock (AMNH). Type locality: Guyana.

Description. Dorsal surface dark- to reddish-castaneous (reddish on apical 3/4 of corium) (fig. 7). Head entirely dark-castaneous, almost black; wrinkles conspicuous. Punctures densely distributed. Antennae black. Second antennal segment more than one and a half longer than first and as long as third; fourth and fifth lacking on the holotype. Pronotum dark-castaneous. Punctures coarse, moderately distributed, more concentrated behind cicatrices; finer punctures, densely distributed, before cicatrices. Scutellum dark-castaneous; coarse punctures on basal 2/3, more concentrated along lateral margins. Corium reddish, except on basal fourth, dark-castaneous, almost black; a yellowish callus near and entad apex of radial vein. Ventral surface of thorax darkcastaneous, almost black; fine punctures regularly distributed on propleura, mesoepisternum and metaepimerum. Ostiolar rugae reaching about half the distance between inner margin of ostiolum and lateral margin of metapleura. Metasternal carina, in profile, strongly elevated anteriorly. Legs dark-castaneous, almost black, yellowish-castaneous at apex of femora and base of tibiae. Connexival segments dark-castaneous, with fine punctures regularly distributed. Venter uniformly dark-castaneous, almost black. Punctures fine, distributed more densely among spiracles, and a narrow, impuctated medial stripe. Spiracles black.

Male genitalia. Pygophore subquadrangular, slightly wider apically. Lobes of dorsal rim little projected posterad, rounded at apex. Genital cup processes moderately visible dorsally (figs. 17, 27). Parameres with wide apex, laterally directed (fig. 47). Ventral rim openly V-like (fig. 37).

Measurements $(n=1)$. Head length 1.96, width 3.08; interocular distance 1.52; anteocular length 0.60; length of antennal segments: I - 0.84; II - 1.36; III - 1.36; IV and V - lacking; pronotal length 4.34, width 10.19; scutellar length 6.68, width 6.51; corium length 9.85; abdominal width 11.02; total length 15.70 .

Distribution. Guyana.

Comments. Brachystethus schuhi shares with its sister species B. cribrus the yellowish callus on corium, but can be distinguished by the following characters: venter uniformly castaneous, dark-castaneous coxae and genital cup processes moderately visible dorsally. Brachystethus schuhi is also closely related to B. signoreti and $B$. rubromaculatus (BARCELLOS \& GRAZIA, 2003), being distinguished from the the former by the callus on corium and male genitalia, and from the latter by the yellowish apex of femora and base of tibiae. 


\section{Brachystethus tricolor Bolívar, 1879}

(Figs. 8, 18, 28, 38, 48, 59)

Brachystethus tricolor Bolívar, 1879:138; Lethierry \& SEVERIN, 1893:187 (cat.); KirKaLdy, $1909: 152$ (cat.); Froeschner, 1981:69 (cat.).

Type material. Lectotype $\sigma$, here designated, labeled: a) Baeza (Ecuador), 4/mar 20/abr/1865, F. Martinez; b) Expedición al Pacífico (1862-1865); c) M.N.C.N., Madrid; d) Brachystethus tricolor Bol.?; e) sintipo? (MNCS); paralectotypes $\checkmark$, ६, both labeled: a) Martinez Baeza/ 481; b) Baeza (Ecuador), 4/mar - 20/abr/1865, F. Martinez; c) Expedición al Pacífico (1862-1865); d) M.N.C.N., Madrid; e) Brachystethus tricolor Bol.?; f) sintipo? (MNCS). Type locality: Baeza, Ecuador. ("Baeza Americae Equatorialis").

Description. Dorsal surface black, except corium and, in some specimens, part of pronotum, reddish (fig.8). Head entirely black, with conspicuous wrinkles. Mediumsized punctures moderate to sparsely distributed. Antennae black; fourth antennal segment cylindrical. Second antennal segment slightly longer than first and about 3/4 as long as third; third slightly more than half of fourth; fifth the longest, a little less than one and a half as long as fourth. Pronotum entirely black, or, in some specimens, with about basal $2 / 3$ reddish. Anterolateral margins entirely black or with a thin subcaloused, yellowish border. Medium-sized punctures concentrated before cicatrices. Some specimens with very coarse punctures on basal $2 / 3$, decreasing in size posterad. Scutellum black, sometimes slightly reddish in the middle. Coarse punctures, in variable density, decreasing in size toward apex. Remaining punctures medium-sized to fine, distributed all over surface of scutellum. Corium reddish, except along costal margins, with a narrow black stripe variable in length, and a little black spot, irregular-shaped, entad to apex of radial vein. Yellowish callus near and entad apex of radial vein, lacking in some specimens. Costal margins, in some specimens, with a thin, subcaloused yellowish border, evanescent posterad. Ventral surface of thorax black, sometimes reddish-castaneous on propleura and near coxae. Punctures concolor. Ostiolar rugae short, extending by about $1 / 3$ of distance between inner margin of ostiolum and lateral margin of metapleura. Metasternum, in profile, little elevated anteriorly; transversal sulcus in the middle. Legs black, coxae reddish-castaneous. Connexivum mostly black; in some specimens, a reddish stripe of variable width along inner margin. Venter red, with rounded black spots around each spiracle and one black continuous stripe along lateral margins, variable in width, occupying one fourth to half the distance between spiracles and lateral margin of abdomen. Some specimens present, on sternites III to VI, entad to spiracles, elliptical black spots, variable in size. Anterior and posterior margins of sternites occasionally with blackish stripes. Punctures sparse, fine and concolor with venter, more concentrated over black spots entad to spiracles. Spiracles black.

Male genitalia. Pygophore quadrangular, equally wide at apex and base. Lobes of dorsal rim conspicuous, strongly projected posterad, rounded at apex. Genital cup processes little visible dorsally (figs. 18, 28). Parameres with acute apex, turned toward tenth segment (fig. 48). Ventral rim not so openly V-like as in other species of the genus (fig. 38).

Measurements $(\mathrm{n}=4)$. Head length $1.80(1.72-1.87) \pm 0.06$, width $2.71(2.61-2.77)$ \pm 0.07 ; interocular distance $1.46(1.37-1.56) \pm 0.08$; anteocular length $0.60(0.56-0.62) \pm$ 0.03 ; length of antennal segments: I - $0.97(0.94-1.01) \pm 0.03$; II - 1.12 (1.05-1.20) \pm 0.06 ; III $-1.44(1.25-1.56) \pm 0.14 ; \mathrm{IV}-2.53(2.50-2.56) \pm 0.03 ; \mathrm{V}-3.44(3.39-3.48) \pm 0.06$; pronotal 

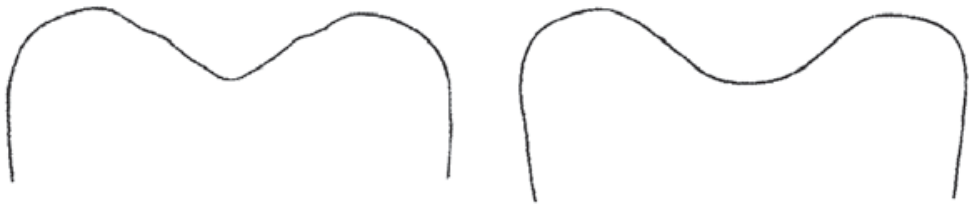

34
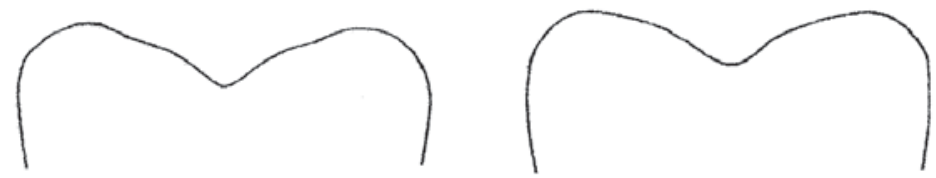

36
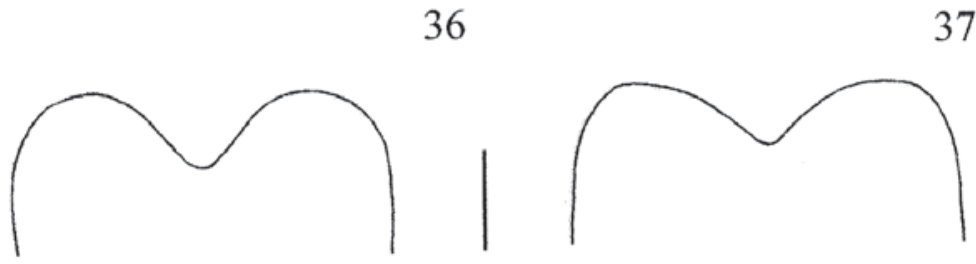

38

39
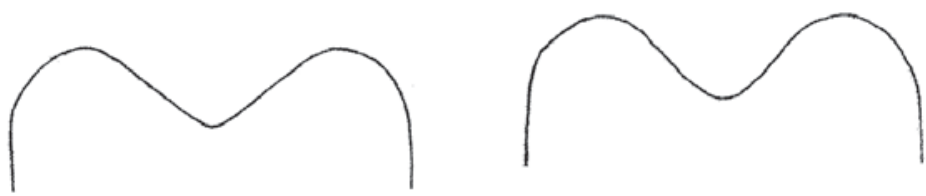

40

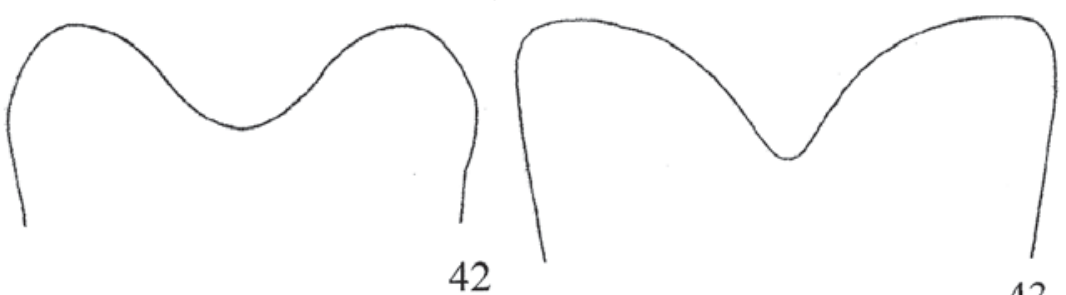

43

Figs. 34-43. Pygophore, ventral rim, ventral: 34, Brachystethus rubromaculatus; 35, B. signoreti; 36, B. cribrus; 37, B. schuhi; 38, B. tricolor; 39, B. vexillum; 40, B. geniculatus; 41, B. coxalis; 42, B. improvisus; 43, B. vicinus. Scale, $1 \mathrm{~mm}$.

length 3.86 (3.34-4.09) \pm 0.35 , width 9.14 (8.52-9.69) \pm 0.50 ; scutellar length 5.95 (5.52-6.35) \pm 0.41 , width $5.72(5.36-6.01) \pm 0.28$; corium length $8.64(8.52-8.77) \pm 0.18$; abdominal width $10.23(9.69-10.69) \pm 0.46$; total length $14.15(13.53-14.86) \pm 0.55$.

Female genitalia. Posterior borders of gonocoxites 8 in an obtuse angle near limit between laterotergites 9 and gonocoxites 9 . Sutural borders of gonocoxites 8 parallel and contiguous, except at apex (fig. 59).

Measurements $(\mathrm{n}=1)$. Head length 1.87 , width 2.89 ; interocular distance 1.56; 
anteocular length 0.62 ; length of antennal segments: I - 1.17; II - 1.29; III - 1.40; IV - 2.77; V - 3.59; pronotal length 3.84 , width 9.69 ; scutellar length 6.24 , width 6.00 ; corium length, not measured; abdominal width 11.19; total length 15.20 .

Material examined. ECUADOR, o, "71", E. de Ville col., "Coll. R.I. Sc. N.B." (DARC). PERU, Ucayali: Aguaytía (295m, low jungle), 3, 13.IX.1946, F. Woytkowski (USNM); Huánuco: Chinchao (25 Km below Carpish, 2500m), 3, 13.IX.1946, F. Woitkowski (AMNH).

Distribution. Ecuador, Peru (Ucayali, Huánuco).

Comments. Brachystethus tricolor is the sister species of B. vexillum, with which shares two synapomorphies and one homoplasy, respectively: venter predominantly red, metasternal carina transversely sulcated and ostiolar rugae short (BARCELLOS \& GRAZIA, 2003). These characters also separate both species from the remaining species of the genus. Brachystethus tricolor can be distinguished from B. vexillum by dorsal color with red spots, and quadrangular shape of pygophore.

\section{Brachystethus vexillum Breddin, 1903}

(Figs. 9, 19, 29, 39, 49, 60, 69)

Brachystethus vexillum Breddin, 1903:371; Bergroth, 1908:178; Kirkaldy, 1909: 152; Pirán, 1955:337. Daimonocoris bolivianus Kormilev, 1951:38; PIRÁN, 1955:337 (syn.).

Type material of B. vexillum. Holotype os, examined, labeled: a) Bolivia; b) Brachystethus vexillum Bredd; c) coll. Breddin; d) Holotypus; e) Dtsch. Entomol. Institut Berlin; f) HT vexillum (DEIC). Type locality: Bolivia.

Description. Dorsal surface black, with yellowish stripes on corium (fig. 9). Head black, with well-marked wrinkles. Punctures moderately distributed, restrict to base of jugae and tylus. Antennae black; fourth antennal segment cylindrical. First antennal segment about 3/4 longer than second, third slightly longer than second, fourth almost twice longer than third and about 3/4 the length of fifth, the longest. Pronotum black, with fine punctures regularly distributed and coarse punctures sparse behind cicatrices. Before cicatrices, medium-sized punctures densely distributed. Anterolateral angles yellowish at apex. Scutellum black, with coarse punctures sparse, more frequent beside lateral margins of basal half. Corium black, without callus, with two yellow stripes: one on clavus, adjacent to claval suture and another occupying $2 / 3$ ectad to radial vein, becoming broader apically toward clavus. Legs dark reddish-castaneous to black. Ventral surface of thorax dark reddish-castaneous to black; medium-sized punctures, concolor. Ostiolar rugae short, reaching $1 / 3$ of distance between inner margin of ostiolum and lateral margin of metapleura. Metasternal carina with conspicuous transversal sulcus; in profile, little elevated anteriorly. Connexivum black, inner superior angle of each segment orange, segments finely punctured. In some specimens, the orange area extends along inner lateral margin of seventh-segment. Venter red, with black spots on lateral thirds, as follows: one near lateral margin of each segment, becoming broader posterad, other small, around spiracles. On sternites III to VI, also present, entad to others, a larger spot, sub-elliptical, close to spiracle. Coarse punctures more concentrated on lateral thirds, over these spots. Spiracles black.

Male genitalia. Pygophore globose, apex and base equally wide. Lobes of dorsal rim conspicuous, strongly projected posterad, rounded at apex. Genital cup processes little visible dorsally (figs. 19, 29). Parameres with acute apex, anteriorly directed (fig. 49). Ventral rim openly V-like (fig. 39). 
Measurements $(n=3)$. Head length $1.73(1.68-1.76) \pm 0.05$, width $2.56(2.52-2,60) \pm$ 0.04 ; interocular distance $1.49(1.44-1.52) \pm 0.05$; anteocular length 0.64 ; length of antennal segments: I - $0.85(0.80-0.88) \pm 0.05$; II - 1.17 (1.12-1.20) \pm 0.05 ; III - $1.44(1.28-1.52) \pm 0.14$; IV - $2.59(2.48-2.72) \pm 0.12 ; \mathrm{V}-3.68$; pronotal length $3.70(3.59-3.84) \pm 0.13$, width 8.63 (8.52-8.85) \pm 0.19 ; scutellar length 5.87 (5.85-5.93) \pm 0.05 , width 5.29 (5.18-5.43) \pm 0.13 ; corium length $8.24(8.02-8.52) \pm 0.26$; abdominal width $10.08(9.69-10.35) \pm 0.35$; total length $13.64(13.03-14.28) \pm 0.63$.

Female genitalia. Sutural borders of gonocoxites 8 parallel, except on apical fifth. Posterior borders convex on outer half and sub-retilineous on inner half (fig. 60). Gonocoxites 9 almost three times wider across apex of arms than long medially; anterior border openly U-like, moderately excavate at middle. Length of arms of gonocoxites 9 equal to $1 / 6$ of plate width. Thickening of vaginal intima digitiform, elongated. Ductus receptaculi thin, one and a half longer before than after vesicular area. Capsula seminalis digitiform. Annular crests sub-parallel, turned toward vesicular area (fig. 69).

Measurements $(n=9)$. Head length $1.93(1.80-2.08) \pm 0.08$, width $2.77(2.72-2.84) \pm$ 0.05; interocular distance $1.60(1.48-1.68) \pm 0.06$; anteocular length $0.69(0.64-0.72) \pm 0.04$; length of antennal segments: I - $0.91(0.84-0.96) \pm 0.04$; II - $1.24(0.96-1.36) \pm 0.13$; III - 1.56 $(1.36-1.88) \pm 0.16 ;$ IV $-2.75(2.60-2.96) \pm 0.13 ; \mathrm{V}-3.58(3.44-3.68) \pm 0.09$; pronotal length 4.12 (3.84-4.34) \pm 0.15 , width 9.65 (9.27-10.02) \pm 0.25 ; scutellar length 6.74 (6.51-7.01) \pm 0.17 , width $5.97(5.68-6.43) \pm 0.21$; corium length $9.32(8.93-9.85) \pm 0.27$; abdominal width $11.44(11.19-11.52) \pm 0.13$; total length $15.56(14.95-16.70) \pm 0.56$.

Material examined. PERU, Cuzco: Machu Picchu, $\subsetneq$, 28.XI.1982, E.R. Ross col., California Academy of Sciences (DARC); (over ruins, 2600-2800m), 2 3, 4 \&, 1.VII.1964, B. Malkin (MZSP); f, 7.V.1965 (arbusto) (MZSP); $\subsetneq$, 1942, Weyrauch (USNM); Prov. Paucartambo, Pillahuata (2800m), 了, 2 †, 19.XII.1952, F. Woytkowski (USNM); Marcapata, †, "Coll. I.R.Sc. N.B." (DARC). BOLIVIA, Yungas de Palmar (2000m), ₹, R. Zischka (USNM); Cochabamba: Chapare prov., Alto Palmar (110m), 2 3, 2 f, XI.1961 (DARC).

Distribution. Peru (Cuzco), Bolivia (Cochabamba).

Comments. Brachystethus vexillum is closely related to B. tricolor. The dorsal color pattern, with yellow stripes, and the globose pygophore distinguish this species from B. tricolor.

\section{Brachystethus geniculatus (Fabricius, 1787)}

(Figs. 1, 10, 20, 30, 40, 50, 61, 70)

Cimex geniculatus FABricius, 1787:293; 1794:115; GMELIn, 1793:2151.

Edessa geniculata; FABRICIUS, 1803:153.

Edessa (Brachystethus) marginatus LAPORTE, 1832:63, pl.54, fig.8.

Edessa (Brachystethus) marginata; BurmeIster, 1835:356 (syn.).

Edessa circumscripta BURMEISTER, 1835:356.

Brachystethus marginatus; SPINola, 1837:343; Amyot \& SERville, 1843:161; Herrich-SchÄFFER, $1845: 2$ (syn.); Dallas, 1851:336; Signoret, 1851:344; Herrich-SchäFFer, 1853:310; StÅL, 1860:28; $1872: 48$ (syn.); WALKER, 1868:455.

Brachystethus circumscriptus; Amyot \& Serville, 1843:161 (syn.); Herrich-Schäffer, 1845:2, fig. 789; Dallas, 1851:336; MAYr, 1866:68; Walker, 1868:455; StÅL, 1872:48 (syn.).

Brachystethus geniculatus; MAYR, 1866:68; STÅL, 1868:38 (redescription); 1872:48; BERG, 1892:11; Lethierry \& Severin, 1893:187 (cat.); Kirkaldy, 1909:152 (cat.); Pennington, 1920:10 (distr.); Rufinelli \& PirÁn, 1959:12 (distr.); BuCKup, 1961:9; Silva et al., 1968:54 (host plant); GraZIA-VIEIRA \& Casini, 1973:58 (distr.); GraZia, 1977:164-165; Froeschner, 1981:69 (cat.); Link \& GraZia, 1987:118 (distr.). 

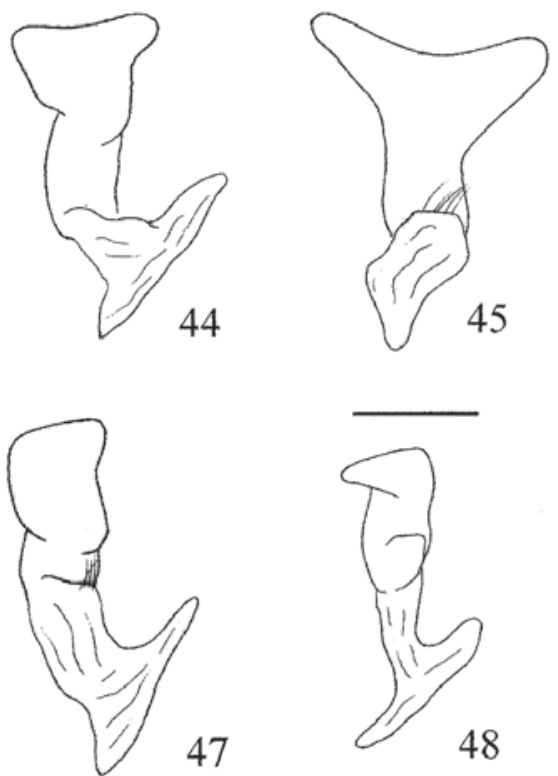

47

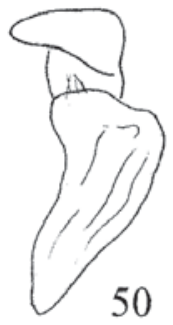

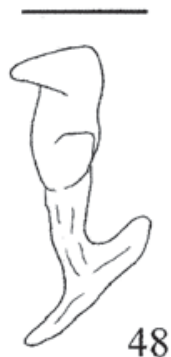

48

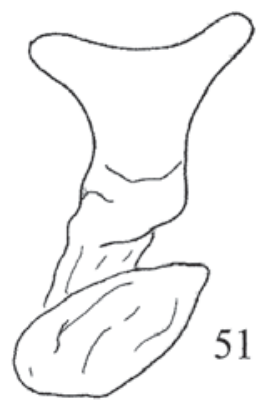

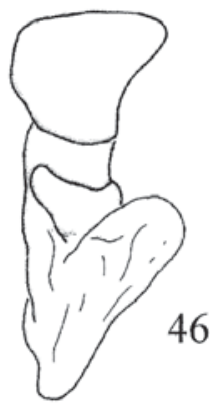

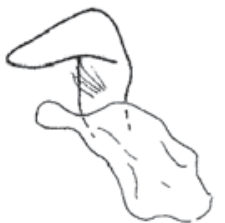

49

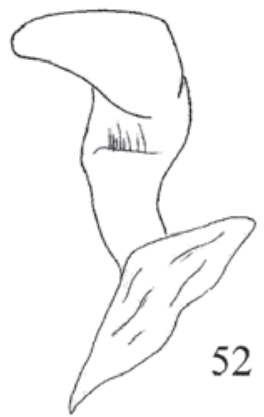

Figs. 44-52. Left paramere, posteroventral: 44, Brachystethus rubromaculatus; 45, B. signoreti; 46, B. cribrus; 47, B. schuhi; 48, B. tricolor; 49, B. vexillum; 50, B. geniculatus; 51, B. improvisus; 52, B. vicinus. Scale, $0.5 \mathrm{~mm}$.

Type material. Holotype $\sigma$, labeled: geniculatus (ZMUC). This specimen, sent as one among three syntypes of $C$. geniculatus, is the only one whose characters fit with the original description, thus it is here designated as holotype. The second specimen fits the description of Cimex cribrum, as formerly commented, and the third fits the diagnostic characters of B. coxalis Breddin. Type locality: "Cajennae". Most of the specimens studied were collected in eastern and southern Brazil, with Bahia State as its northern limit. Literature also indicates the occurrence of B. geniculatus in Uruguay. As at Fabricius time the label data were quite vague, and Caiene was indeed a site of departure and arrival of the expeditions, we consider that Caiene does not actually correspond to the collecting locality of the specimen. 
Description. Dorsal surface castaneous to dark-castaneous (fig. 10). Head castaneous to dark-castaneous, with little conspicuous wrinkles. Punctures on medial region and semi-circular stripes densely distributed. Remainder of jugae moderately to sparsely punctured, depending on the specimen. Jugae with yellow stripe on outer lateral margins, evanescent anterad. Antennae reddish-castaneous; second antennal segment one and a half longer than first; third slightly longer than second; fourth one and a half longer than third and 3/4 of the length of fifth, the longest. Fourth antennal segment cylindrical. Pronotum castaneous to dark-castaneous, with yellowish stripe on anterolateral margins, and less conspicuous on the anterior margin. Punctures fine, darkcastaneous to black, regularly distributed, except on cicatrices. Among these punctures, present larger ones, black, more concentrated before cicatrices and behind them, where they achieve their maximum size. Scutellum with color similar to the rest of dorsal surface, with coarser punctures on anterior half (fig. 1). Remaining punctures fine, homogeneously and densely distributed. Corium castaneous, with a yellowish callus, internally and near to apex of radial vein. Legs uniformly reddish-castaneous. Ventral surface of thorax castaneous, punctured with dark-castaneous. Ostiolar rugae extending by about $2 / 3$ of distance between inner margin of ostiolum and lateral margin of metapleura. Metasternal flat in profile. Connexivum castaneous, lateral outer $1 / 6$ of each segment with subcaloused yellowish stripe. Entad to this border, punctures fine, densely and homogeneously distributed. Venter castaneous, bordered with a thin subcaloused, yellowish stripe. Medium-sized, concolor punctures densely distributed, except on a narrow medial stripe. Spiracles yellowish-castaneous, bordered with black.

Male genitalia. Pygophore subquadrangular, slightly wider at apex. Lobes of dorsal rim conspicuous, moderately projected posterad, sub-acute at apex. Genital cup processes little visible in dorsal view (figs. 20, 30). Parameres with acute apex, turned anterad (fig. 50). Ventral rim openly V-like (fig. 40).

Measurements $(n=10)$. Head length $1.95(1.76-2.12) \pm 0.13$, width $2.93(2.72-3.16) \pm$ 0.16; interocular distance 1.61 (1.52-1.72) \pm 0.07 ; anteocular length $0.67(0.56-0.80) \pm 0.07$; length of antennal segments: I - $0.82(0.76-0.88) \pm 0.04$; II - $1.33(1.04-1.68) \pm 0.22$; III - 1.60 $(1.36-1.84) \pm 0.16 ;$ IV $-2.57(2.32-3.04) \pm 0.26 ; \mathrm{V}-3.28(2.88-3.92) \pm 0.37$; pronotal length 4.08 (3.67-4.68) \pm 0.37 , width $9.82(8.68-11.02) \pm 0.81$; scutellar length $6.66(6.01-7.52) \pm$ 0.57 , width $6.30(5.68-7.01) \pm 0.49$; corium length $8.85(8.18-9.69) \pm 0.52$; abdominal width $10.77(9.52-12.02) \pm 0.83$; total length $14.89(13.36-16.37) \pm 0.99$.

Female genitalia. Gonocoxites 8 rounded to sub-quadrangular. Sutural borders of gonocoxites 8 parallel and posterior borders bissinuated, in almost right angle over laterotergites 9 (fig. 61). Gonocoxites 9 more than three times wider across apex of arms than long medially; anterior border openly U-like, arms length equal to 1/4 of plate width. Thickening of vaginal intima digitiform. Ductus receptaculi thin after vesicular area and slightly shorter than before to this area. Capsula seminalis globose. Annular crests convergent (fig. 70).

Measurements $(n=10)$. Head length $2.08(1.92-2.24) \pm 0.10$, width $3.07(2.84-3.40) \pm$ 0.17 ; interocular distance 1.72 (1.60-1.88) \pm 0.09 ; anteocular length $0.70(0.60-0.80) \pm 0.07$; length of antennal segments: I- $0.90(0.80-0.96) \pm 0.05$; II - $1.40(1.20-1.84) \pm 0.20$; III - 1.70 $(1.44-2.08) \pm 0.20 ;$ IV $-2.73(2.36-3.36) \pm 0.33 ; \mathrm{V}-3.38$ (2.96-4.08) \pm 0.43 ; pronotal length 4.43 (4.01-5.18) \pm 0.37 , width $10.48(9.52-12.02) \pm 0.86$; scutellar length $7.41(6.85-8.68) \pm$ 0.61 , width $6.79(6.35-7.77) \pm 0.49$; corium length $9.74(8.85-11.36) \pm 0.80$; abdominal width $11.85(10.69-13.28) \pm 0.86$; total length $16.34(15.11-18.54) \pm 0.99$. 

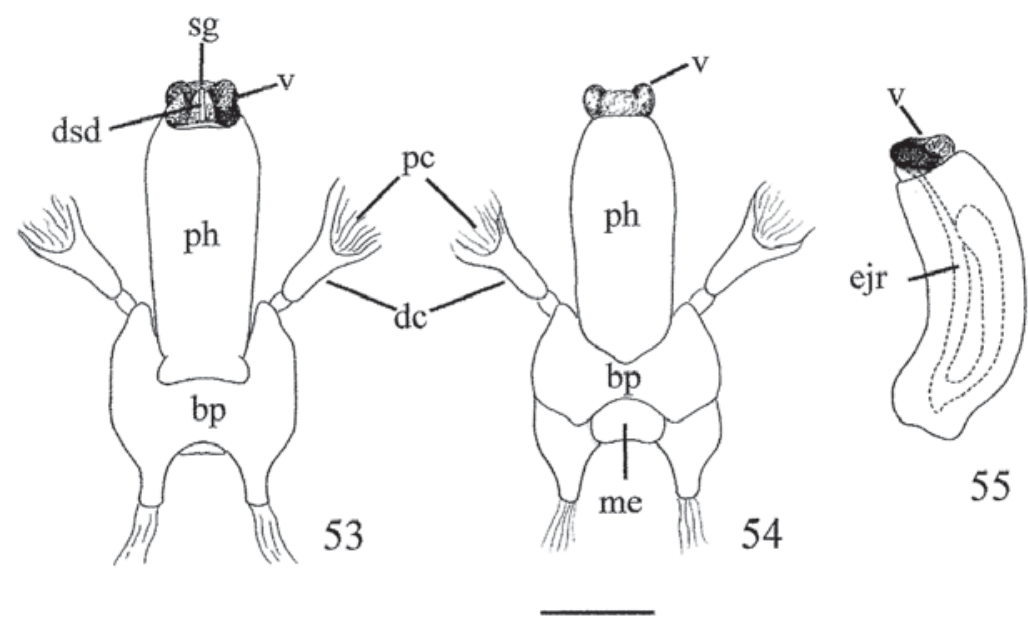

Figs. 53-55. Brachystethus rubromaculatus. Phallus, respectively dorsal, ventral and lateral (bp, basal plate; dc, dorsal conective; dsd, ductus seminis distalis; ejr, ejaculatory reservoir; me, membranblase; pc, processus capitati; ph, phallotheca; $\mathrm{sg}$, secondary gonopore; v, vesica). Scale, $1 \mathrm{~mm}$.

Material examined. BRAZIL, ठ, Pres. by Perth Museum (BMNH 1953-629); $\subsetneq$, Blackmore col., 74, Pres. by Perth Museum (BMNH 1953-629); Chapada, ơ, acc. 23739 (AMNH); ठ, XII, acc. 23739 (AMNH); Bahia: Ilhéus, 3 ơ, Ф, III.2000, C.A.L. Guimarães (in Attalea funifera) (UFRG); Minas Gerais: Poços de Caldas (Morro São Domingos), ơ, f, 19.XII.1967, 29.II.1968, J. Becker, O. Roppa \& O. Leoncini (MNRJ); Barbacena, §, H. Ebert (AMNH); Goiás: Pirineus, \&, 2.II.1962, J. \& B. Bechyné (MPEG); Espírito Santo: Linhares, Ő, ㅇ, I.1962, Claudionor Elias (DZUP); Santa Tereza, ક, 24-31.IV.1967, C. \& C.T. Elias (DZUP); ‡, 18-23.XII, C. Elias (DZUP); B. Guandu, హ, 17.V.1970, C. \& C.T. Elias (DZUP); Rio de Janeiro: o', IX, 23734 (AMNH); Rio de Janeiro (Corcovado), 3 f, IX, X.1961, Seabra \& Alvarenga (DZUP); o', 15.IX.1961, J.S. Moure, Alvarenga \& Seabra (DZUP); (Estr. Gávea), ठ , 10.VII.1942, Pero M. Oliv. (UFRG); (Botafogo), §, XI.1942, Pero M. Oliv. (UFRG); (Alto da Boa Vista), §, X.1943, R. Más (UFRG); (Tijuca), o, 13.IV.1943, R. Braga (UFRG); Itatiaia (816m), Ғ, 1.I.1933, N.B. Fagundes (UFRG); São Paulo: $\subsetneq$, $20 . I I I .71$ (DZUP); f, 4.VIII.1974, C. Bordin (UFRG); Barueri, 6 of, 5 f, 02, 20.VII, 12.VIII, 12.XI.1954, 18.VII, 6.XII.1955, K. Lenko (MNRJ); Angatuba, హ, XII.1922, Azevedo Marques, Inst. Biologia Entomologia Agrícola Rio de Janeiro 3.492 (UFRG); Paraná: Xaxim, o, 1941, A. Souza (MNCE 13013); Imbaú, F, IX.1942; (MNCE); Pinhão (Rio Iguaçu, próximo à Usina Hidrelétrica de Segredo), 2 హ, \&, VII.1992, G. Skuk (MNCE); Alexandra, đ, 10.X.1970, Moure \& Mielke (DZUP); Ponta Grossa, o, 3 §, XI.1945, 22.II, 23.IV.1946 (MNCE); (pedreira), హ, XII.1943 (DZUP); Piraquara, 3, 14.IV.1983, N. Gonçalves (MNCE); (mananciais da serra, $( \pm 800 \mathrm{~m}$ alt.), §, S.L. Bonatto (UFRG); Curitiba, \&, IX.1945 (MNCE); f, 16.II.1966, C. Ext. DZUFP (DZUP); (Fonte Ahú), f, XII.1936, Claretiano (MNCE); Praia do Leste, ơ, 26.V.1979, I.W. Forno (DZUP); Santa Catarina: ơ, I.1946, A. Maller, Frank Johnson

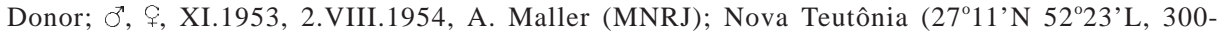
500m), o, II.1981, Fritz Plaumann (DZUP); Corupá (Hansa Humbolt), §, II.1948, A. Maller col., Frank Johnson Donor (AMNH); Florianópolis (Canasvieiras), §, Ғ, I.1946, C. Goffergé (MNCE 10258, 10257); Rio Grande do Sul: ơ, \&, 6.XII.1973, R. Wagner Hanisch \& A. Ruszczyk (UFRG); 3, 17.XII.1973, E. Heinrichs (UFRG); ₹, 19.III.1974, R.A. Ilana (UFRG); Derrubadas (Parque Florestal Estadual do Turvo), 3 o', 3 \&, 3.IX.1982, 21-25.V.1984, S.L. Bonatto (UFRG); Porto Alegre, \&, V.1955, O.P.H. (em Ayrax leprosum C. de vaca) (UFRG); (Morro Santana), 3 ơ, 10 \&, 13.VIII.1982, S. Bonatto (em quiescência) (UFRG); ơ, 4 f, 15.IX.1983, M. Becker (em quiescência em bromeliáceas) (UFRG); (Reserva do Lami), f, 12.II.1992, A.B.P. Santos (UFRG); (Petrópolis), ł, 13.XII.1982, G. 
Bond, L. Buckup (UFRG 271); (Vila Manresa),, 16.II.1965, Pe. Buck (UFRG 6306); (Belém Novo), 3, 2 Ғ, 17.IV.1955, M.R. Oliveira (UFRG); $\{$, 11.IV.1981, R.P. Mocellin (UFRG); Santa Maria, f, 10.IV.1981, A. Stringhini (UFRG); São Sepé, f, 17.V.1981, C.R. Kelling (UFRG); З, 27.IV.1989, Link \& Costa (UFRG); Guaíba (E.E. Guaíba), §, 15.IV.1974, E. Heinrichs (UFRG); Viamão, f, 18.IX.1993, L.A. Campos (UFRG); Pelotas, $\subsetneq$, 10.II.1941 (UFRG); $\subsetneq$, 14.IV.1961, C.M. Biezanko (BMNH); 2 o, f, 16.XII.1961, 05.IV.1963, 7.IV.1966, C.M. Biezanko (BMNH 1965-175, 1963375, 1966-318); 2 f, 14.V.1967, C.M. Biezanko (AMNH); 2 §, 2 f, 14.V.1967, J. L. Mantovani \& Biezanko (BMNH 1967-265). BOLIVIA, Santa Cruz: San Francisco, ð, 1895, A. Borelli (MRSN). PARAGUAY, Itapúa: Hohenau, ơ, III.1955, J.L. Stehlik (BMNH 1966-601). ARGENTINA, Buenos Aires: Buenos Aires, ơ, Hoefel, Coll. J. Stehlik (BMNH 1964-449); ठ, II.1953, Hoefel, Coll. J. Stehlik (BMNH 1966-601). No data: 2 (AMNH, UFRG); §, P.R. Uhle col. (AMNH).

Distribution. Brazil (Bahia, Minas Gerais, Goiás, Espírito Santo, Rio de Janeiro, São Paulo to Rio Grande do Sul), Bolivia (Santa Cruz), Paraguay (Itapúa), Argentina (Buenos Aires).

Comments. Brachystethus geniculatus is the sister group of the clade formed by B. coxalis, B. improvisus and B. vicinus, sharing with them the yellowish border of pronotum and the callus on corium (BARCELlos \& GRAZIA, 2003). Brachystethus geniculatus is very similar to $B$. coxalis, by the parameres, with acute apices, and by the metasternal carina, flat in profile, contrasting with the carina strongly elevated of the other two species. Brachystethus geniculatus is differenciated from B. coxalis, B. improvisus and $B$. vicinus by the uniform color pattern of legs.

\section{Brachystethus coxalis Breddin, 1904}

(Figs. 11, 21, 31, 41, 62)

Brachystethus coxalis Breddin, 1904:178; Bergroth, 1908:178; KirKaldy, 1909:152 (cat.).

Type material. Lectotype $\sigma$, here designated, labeled: a) Amazonas, Peru; b) Brachystethus coxalis Bredd; coll. Breddin; d) Syntipus; e) DEI Eberswalde; f) 1 ST coxalis (DEIC). Breddin described the species based on two specimens, one male and one female. Only the male syntype was found. Type locality: Peru (Amazonas) and Panama (Chiriquí).

Description. Dorsal surface dark-castaneous to black, with a marginal yellow stripe (fig. 11). Head dark-castaneous, with well-marked wrinkles. Punctures densely distributed. Outer margins of jugae with yellowish stripe, wider at base. Antennae dark-castaneous to black, except the first segment, yellowish. Fourth segment cylindrical. First antennal segment a little more than $3 / 4$ as long as second, third almost twice as long as second, fourth slightly less than one and a half as long as third, fifth lacking. Pronotum darkcastaneous to black, with a broad yellow stripe along anterolateral margins. Punctures fine regularly distributed, with sparse coarse punctures restrict to the area after cicatrices. Before cicatrices, smaller punctures more concentrated along anterior margin of pronotum. Scutellum dark-castaneous to black, with fine punctures regularly distributed. Coarse punctures on basal third, concentrated in 1+1 groups along lateral margins. Punctures slightly smaller, sparse, on basal half. Corium dark-castaneous, with yellow border on basal third of costal margin and with a small yellowish callus near and entad the apex of radial vein. Legs yellowish on coxae, trochanters, base and apex of femora, ventral face and apex of tibiae and tarsi; remaining segments dark-castaneous, almost black. Ventral surface of thorax dark-castaneous to black, with castaneous to black medium-sized punctures. Ostiolar rugae reaching $2 / 3$ of distance between inner margin of ostiolum and 

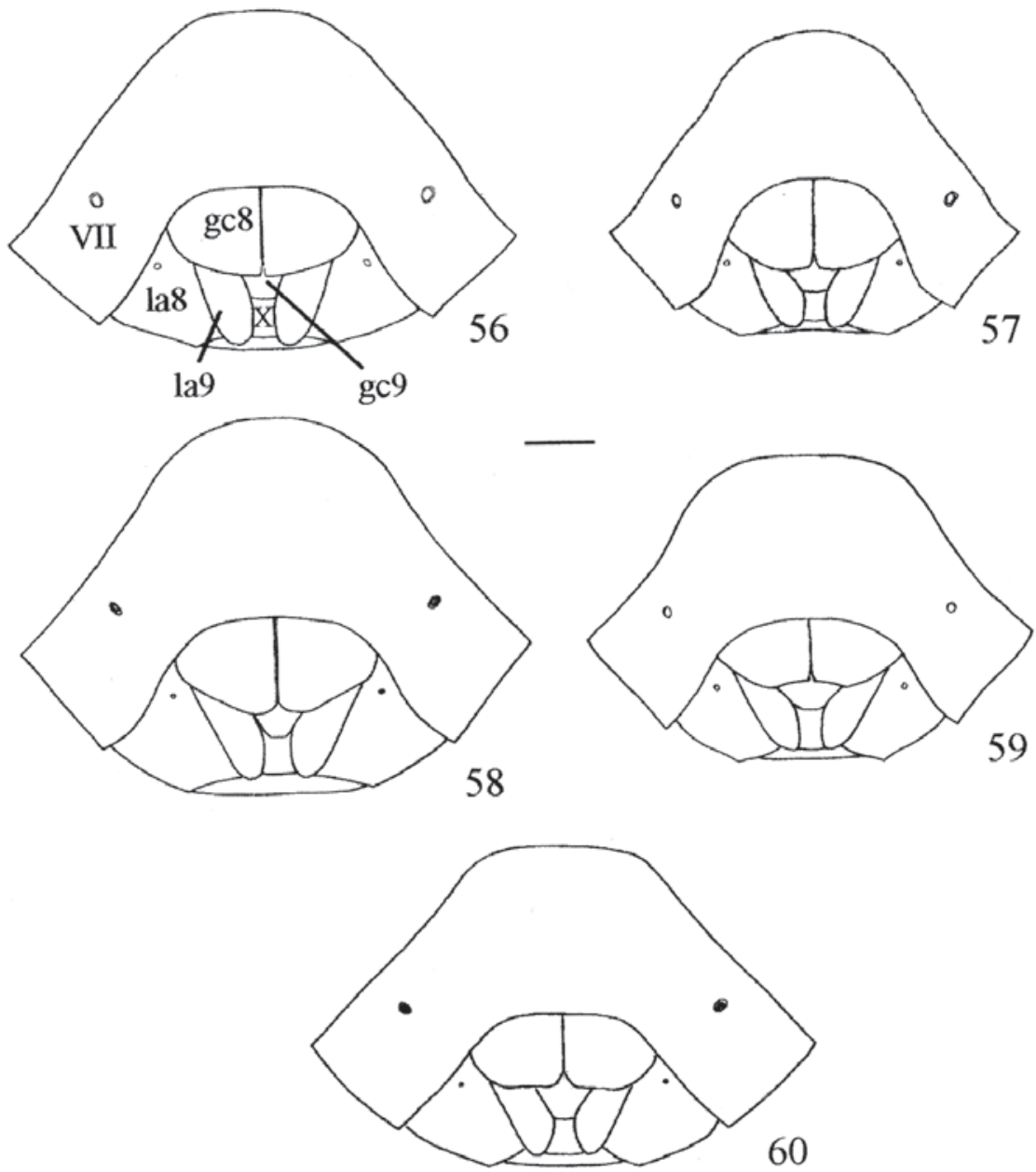

Figs.56-60. Genital plates, ventral: 56, Brachystethus rubromaculatus; 57, B. signoreti; $58, B$. cribrus; 59, B. tricolor; 60, B. vexillum ( $\mathrm{gc} 8$, gonocoxite 8; gc9, gonocoxites 9; la8, laterotergite 8; la9, laterotergite 9; VII, seventh segment; X, tenth segment). Scale, $1 \mathrm{~mm}$.

lateral margin of metapleura. Metasternal carina flat in profile. Connexivum with a subcaloused yellow stripe, scarcely punctured, over outer lateral third of each segment; remaining of connexival segments dark-castaneous, almost black, with punctures minutely and densely distributed. Venter dark-castaneous, with a marginal, subcaloused yellowish stripe. Punctures fine regularly distributed, except on the middle, where they are less frequent.

Male genitalia. Pygophore quadrangular. Lobes of dorsal rim conspicuous, moderately projected posterad, rounded at apex. Genital cup processes not visible in dorsal view. Parameres with acute apex, turned anterad (figs. 21,31). Ventral rim openly V-like (fig. 41). 
Measurements $(n=1)$. Head length 2.03, width 3.00; interocular distance 1.64; anteocular length 0.74; antennal segments - lacking; pronotal length 4.34, width 10.02; scutellar length 6.40, width 6.24; corium length, not measured; abdominal width 11.19; total length 15.87 .

Female genitalia. Posterior borders of gonocoxites 8 forming an almost right angle over laterotergites 9. Sutural borders of gonocoxites 8 parallel (fig. 62).

Measurements $(n=1)$. Head length 2.00, width 3.04; interocular distance 1.60; anteocular length 0.72; length of antennal segments: I - 0.88; II - 1.12; III - 2.00; IV - 2.80; V - lacking; pronotal length 4.18, width 10.02; scutellar length 6.68, width 6.51; corium length 9.52; abdominal width 11.11; total length 15.70.

Material examined. VENEZUELA, Amazonas: Cerro de la Neblina (1880m), f, 11.II.1984, Stupakoff (AMNH). No data, o (ZMUC).

Distribution. Panama (Chiriquí), Venezuela (Amazonas), Peru (Amazonas).

Comments. Brachystethus coxalis is the sister group of B. vicinus and B. improvisus, sharing with them one synapomorphy, the yellowish tarsi, and two homoplastic characters, coxae, and apex of femora and base of tibiae yellowish (BARCELLOS \& GRAZIA, 2003). It can be distinguished from B. vicinus and B. improvisus by the flat metasternal carina, and from the latter also by the shape of parameres, with acute apex.

\section{Brachystethus improvisus Breddin, 1905}

(Figs. 12, 22, 32, 42, 51, 63, 71)

Brachystethus improvisus BREdDIN, 1905:26; Bergroth, 1908:178; KIRKALdy, 1909:152 (cat.).

Type material. Holotype $₹$, examined, labeled: a) Esmerala; b) B. improvisus Bredd./ n. spec. genitalium!; c) coll. Breddin; d) Holotypus; e) DEI Eberswalde; f) 1 HT improvisus (DEIC). Type locality, Venezuela.

Description. Dorsal surface dark-castaneous, with yellow marginal stripe (fig. 12). Head with coarse punctures moderately distributed and conspicuous wrinkles. Outer margins of jugae with yellow stripe, broader at base. Second antennal segment about one and a half longer than first, third slightly longer than second, fourth a little more than one and a half as long as third and about 3/4 as long as fifth, the longest. Fourth antennal segment cylindrical, lacking sulcus. Pronotum with fine punctures, concentrated before cicatrices, and regularly distributed on the remaining pronotum, interposed with a little coarser, sparse punctures. Scutellum with coarse punctures more frequent at basal 2/3. Corium with a yellowish callus near and entad apex of radial vein. Legs castaneous; coxae, base of trochanters and tibiae, apex of femora and tarsi reddish castaneous to yellowish, in extension variable among the specimens. Ventral surface of thorax with castaneous to black medium-sized punctures. Ostiolar rugae reaching about $2 / 3$ the distance between inner margin of ostiolum and lateral margin of metapleura. Metasternal carina strongly elevated anteriorly. Connexivum castaneous to black, with subcaloused yellow stripe ocupying, at least, the outer lateral third of each segment. Punctures fine, more densely distributed entad to this stripe. Venter dark-castaneous, with yellow stripe along lateral margins. Punctures fine, inconspicuous at middle. Spiracles yellowish. Genitalia. Pygophore sub-quadrangular, slightly wider apically. Lobes of dorsal rim conspicuous, strongly projected, rounded at apex. Genital cup processes moderately 

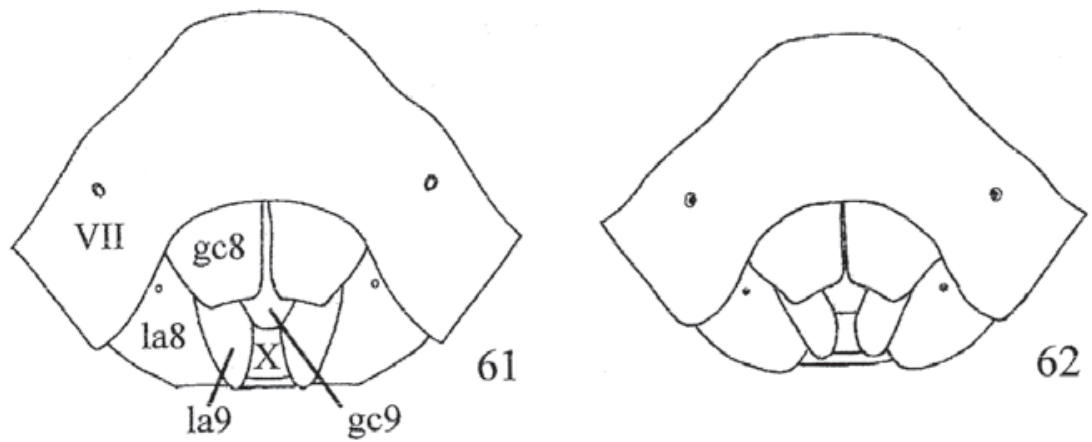

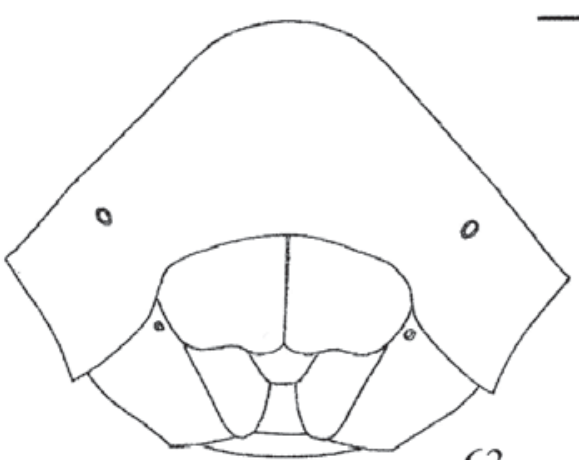

63

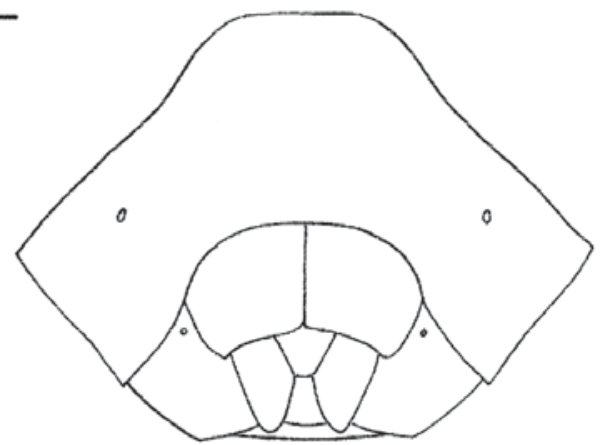

64

Figs. 61-64. Genital plates, ventral: 61, Brachystethus geniculatus; 62, B. coxalis; 63, B. improvisus; 64, B. vicinus (gc8, gonocoxite 8; gc9, gonocoxites 9; la8, laterotergite 8; la9, laterotergite 9; VII, seventh segment; X, tenth segment). Scale, $1 \mathrm{~mm}$.

visible dorsally (figs. 22, 32). Parameres with broad apex, anterolaterally directed (fig. 51). Ventral rim openly U-like (fig. 42).

Measurements $(\mathrm{n}=10)$. Head length $2.09(1.92-2.32) \pm 0.12$, width $3.34(3.16-3.44) \pm$ 0.11 ; interocular distance $1.76(1.68-1.84) \pm 0.07$; anteocular length $0.76(0.72-0.80) \pm 0.04$; length of antennal segments: I - $1.02(0.88-1.36) \pm 0.13$; II - $1.54(1.36-1.76) \pm 0.15 ;$ III -2.06 $(1.68-2.48) \pm 0.25 ; \mathrm{IV}-3.31(2.96-3.60) \pm 0.19 ; \mathrm{V}-4.30$ (4.16-4.40) \pm 0.12 ; pronotal length $4.93(4.51-5.26) \pm 0.21$, width $11.28(10.69-12.02) \pm 0.52$; scutellar length 7.57 (7.01-8.02) \pm 0.35 , width $7.13(6.68-7.68) \pm 0.35$; corium length $10.71(10.35-11.19) \pm 0.26$; abdominal width $12.49(11.36-13.19) \pm 0.61$; total length $17.48(16.37-18.37) \pm 0.71$.

Female genitalia. Posterior borders of gonocoxites 8 sinuate over laterotergites 9 (fig. 63); sutural borders of gonocoxites 8 parallel. Gonocoxites 9 less than three times as wide across apex of arms than long in the middle; anterior border sinuate, little excavated medially. Arms of gonocoxites 9 a little more than 1/5 of plate width. Thickening of vaginal intima digitiform. Ductus receptaculi thin and longer after than before vesicular area. Capsula seminalis ovoid. Annular crests convergent (fig. 71).

Measurements $(\mathrm{n}=10)$. Head length 2.18 (2.00-2.36) \pm 0.12 , width 3.47 (3.36-3.60) \pm 0.07 ; interocular distance $1.82(1.72-1.92) \pm 0.06$; anteocular length $0.79(0.68-0.88) \pm 0.07$; length of antennal segments: I - $1.00(0.80-1.20) \pm 0.11$; II - $1.67(1.44-2.08) \pm 0.19$; III - 1.95 
$(1.68-2.24) \pm 0.18 ;$ IV $-3.37(3.20-3.52) \pm 0.14 ; \mathrm{V}-4.47(4.08-4.72) \pm 0.26$; pronotal length $5.19(4.84-5.51) \pm 0.25$, width 11.85 (10.86-12.86) \pm 0.69 ; scutellar length $8.26(7.68-9.02) \pm$ 0.47 , width $7.54(6.85-8.18) \pm 0.42$; corium length $11.51(10.86-12.53) \pm 0.52$; abdominal width $13.20(12.19-14.20) \pm 0.79$; total length $18.72(17.54-20.21) \pm 0.89$.

Material examined. GUATEMALA, $\sigma$ (AMNH). COSTA RICA, Peralta, $\subsetneq$, V.1935 (BMNH); Guanacaste: Tierras Morenas, Z. P. Tenorio (Rio San Lorenzo, 1050m), హ, 10-20.II.1992 (INBC LN 287800, 427600); Puntarenas: Monteverde, ơ, \&, 4-6.VI.1980, J.E. Wappes col. (DARC); A.C. Arenal, Monteverde (San Luis, 1000-1350m), \&, I.1994, Z. Fuentes (INBC LN 449250, 250850, 2609); (San Luis, Buen Amigo, 1000-1350m), 2 o, V.1994, Grace Fuentes (INBC LN 250850, 449250, 2927); R.B. Monteverde, $\subsetneq$, 24.VIII-15.IX.1992, F. A. Quesada (INBC LN 250850, 449250); Coto Brus (Est. Biol. Las Alturas, 1500m), o, VIII.1991, M. Ramirez (INBC LS 822500, 591300); 了, X.1991, M. A. Zumbado (INBC LS 822500, 591300); †, I.1992, M. Ramirez, G. Mora, F. Quesada (INBC LS 822500, 591300); Ғ, 23.III-2.V.1992, F. Araya (INBC LS 822500, 591300). PANAMA, Chiriquí: $\sigma$, f, 1898, Coll. Noualhier (MNHN); Dist. Renacimiento, Santa Clara (4000'), $\sigma$, 10.IX.82, R. Johnson (AMNH); Fortuna $\left(82^{\circ} 15^{\prime} \mathrm{W} 8^{\circ} 44^{\prime} \mathrm{N}\right)$, §, 3.IV.1977, Henk Wolda (AMNH); (1050m), ‡, 18.V.1978, O'Brien \& Marshall (AMNH); N. Candelas (4000'), §, 24.VIII.82, Stockwell (AMNH); Trail Fortuna to Soledad, ơ, 20.V.1978 (s-facing slope) (AMNH); Repr. La Fortuna, Ғ, 17-21.IX.76, 3200', D. Engleman (DARC); Cerro Azul: north of Tocumén, \&, 7.VI.1958, W.J. Hanson (DARC). COLOMBIA, Cundinamarca: Bogota, $\subsetneq$, Distant Coll. (BMNH 1911-383). BRAZIL, Amazonas: Tefé (Ega), 3, IV.78, I.79, M. de Mathan, Distant Coll. (BMNH 1911-383). BOLIVIA, Santa Cruz: Buena Vista Prov., Tohito (400m), $\subsetneq$, 1956, Franz Steinbach (AMNH). No data: $2 \subsetneq$ (BMNH).

Distribution. Guatemala, Costa Rica (Guanacaste, Puntarenas), Panama (Chiriquí, Cerro Azul), Colombia (Cundinamarca), Brazil (Amazonas), Bolivia (Santa Cruz).

Comments. Brachystethus improvisus is the sister group of B. vicinus (BARCELLOS \& GRAZIA, 2003), sharing with this species the metasternal carina anteriorly elevated and the anterior margin of gonocoxites 9 sinuate, little excavated, besides the general color and the color pattern of legs. The two species are very similar in general appearance, but can be distinguished by male and female genitalia. B. improvisus has the dorsal rim of pygophore with conspicuous lobes, genital cup processes moderately visible in dorsal view and parameres broad at apex; on the females, the posterior border of gonocoxites 8 is sinuate over laterotergites 9 .

\section{Brachystethus vicinus Signoret, 1851}

(Figs. 13, 23, 33, 43, 52, 64, 72)

Brachystethus vicinus Signoret,1851:344; WALKer, 1868:455; StÅL, 1872:48; Distant, 1880:85; LethierRy \& Severin, 1893:187 (cat.); KirKaldy, 1909:152 (cat.); Froeschner, 1981:69 (cat.); Maes, 1994:8 (cat.).

Type material. Lectotype $\sigma$ and paralectotype $\&$, here designated, both labeled: a) Brazil, Coll. Signoret, b) vicinus det. Signoret (NHMW). Type locality: Brazil.

Description. Dorsal surface castaneous to dark-castaneous, with a yellowish marginal stripe (fig. 13). Head castaneous to dark-castaneous, almost black, with wellmarked wrinkles. Punctures moderately distributed. Outer margins of jugae with yellow stripe, wider at base. Antennae dark-castaneous to black, except the first segment, yellowish at base. Fourth antennal segment cylindrical, lacking longitudinal sulcus. Second antennal segment one and a half longer than first and about 3/4 as long as third; third a little more than half as long as fourth, fifth the longest, a little longer than fourth. Pronotum castaneous to dark-castaneous; anterolateral margins with continuous yellow stripe. Medium-sized punctures concentrated before cicatrices and scarce after that, 

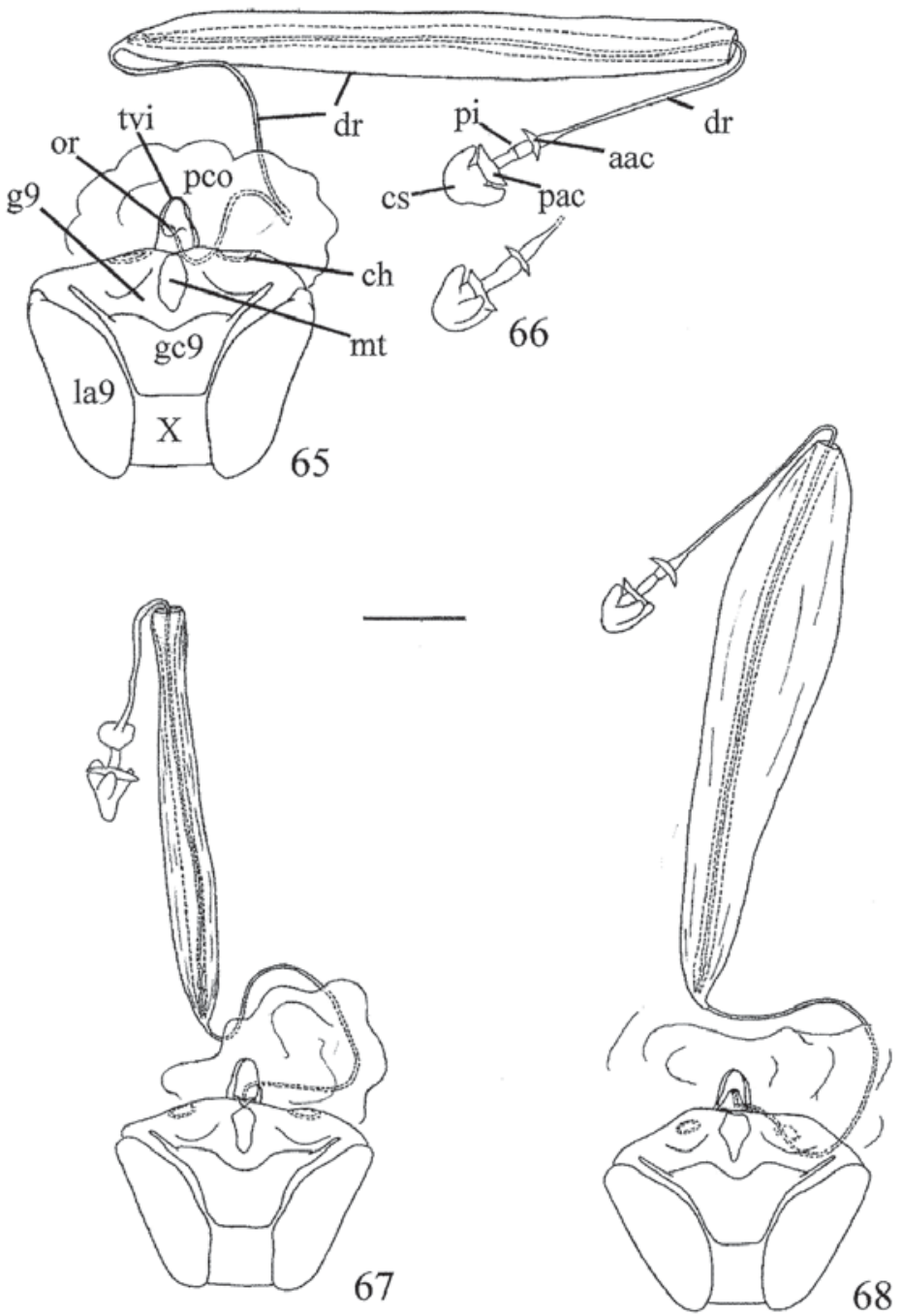

Figs. 65-68. Laterotergites, gonocoxites and gonapophyses of ninth segment, and ectodermical genital ducts, ventral: 65, Brachystethus rubromaculatus; 67, B. signoreti; 68, B. cribrus. Fig. 66, B. rubromaculatus, distal end of receptaculum seminis, dorsal (aac, anterior annular crest; ch, chitinellipsen; cs, capsula seminalis; dr, ductus receptaculi; g9, gonapophyses 9; gc9, gonocoxites 9; la9, laterotergite 9; mt, medial thickening of gonapophyses 9; or, orificium receptaculi; pac, posterior annular crest; pco, pars comunis; pi, pars intermedialis; tvi, thickening of vaginal intima; X, tenth segment). Scale, $1 \mathrm{~mm}$.

more frequent on anterior half of pronotum. Scutellum coarsely punctured, punctures more frequent on anterior half. Costal margins of corium with yellow stripe on anterior third, evanescent posterad. Yellowish callus near and entad apex of radial vein. Legs 
dark-castaneous, except coxae, basal 3/4 of trochanters, apex of femora and base of tibiae, yellowish. Ventral surface of thorax castaneous, with yellow stripe along lateral margins of propleura. Punctures fine, castaneous. Ostiolar rugae reaching 2/3 of distance between inner margin of ostiolum and lateral margin of metapleura. Metasternum, in profile, strongly elevated anteriorly. Connexivum with continuous yellow stripe along outer margin of segments, variable in width. Punctures fine, dense and homogeneously distributed on inner half of each segment. Venter castaneous; lateral margins with a continuous yellow stripe. Punctures fine and concolor, moderately distributed, less frequent in the middle. Spiracles yellowish.

Male genitalia. Pygophore trapezoidal, much wider apically. Lobes of dorsal rim inconspicuous. Genital cup processes little visible dorsally (figs. 23, 33). Parameres long, with acute apex, turned anterad (fig. 52). Ventral rim V-like, deeply excavate medially (fig. 43).

Measurements $(n=4)$. Head length $2.20(2.00-2.34) \pm 0.15$, width $3.53(3.36-3.67) \pm$ 0.14 ; interocular distance $1.85(1.76-1.95) \pm 0.09$; anteocular length $0.79(0.74-0.86) \pm 0.05$; length of antennal segments: I - $1.09(0.96-1.25) \pm 0.14$; II - $1.68(1.56-1.83) \pm 0.11$; III - 2.21 (1.84-2.42) \pm 0.26 ; IV - 3.83 (3.24-4.29) $\pm 0.54 ; \mathrm{V}-4.78$ (4.24-5.62) \pm 0.73 ; pronotal length 5.30 (4.84-5.68) \pm 0.37 , width 12.11 (11.19-12.69) \pm 0.67 ; scutellar length $7.76(7.28-8.24) \pm$ 0.44 , width 7.40 (7.04-7.76) \pm 0.34 ; corium length 10.19 ; abdominal width 13.34 (12.27$14.20) \pm 0.93$; total length $18.83(16.87-20.21) \pm 1.59$.

Female genitalia. Posterior borders of gonocoxites 8 projected in acute angle, over the limit between laterotergites 8 and laterotergites 9; slightly convex on area adjacent to laterotergites 9 and gonocoxites 9 (fig. 64). Sutural borders of gonocoxites 8 parallel and contiguous. Gonocoxites 9 less than twice and a half wider across apices of arms than long medially; anterior border sinuate, little excavate medially. Arms of gonocoxites 9 about $1 / 6$ as long as plate width. Thickening of vaginal intima digitiform. Ductus receptaculi more than one and a half longer before than after vesicular area, equally thickened in both regions. Capsula seminalis globose. Annular crests convergent (fig. 72).

Measurements $(n=6)$. Head length $2.38(2.16-2.50) \pm 0.13$, width $3.66(3.36-3.90) \pm$ 0.22 ; interocular distance $1.95(1.84-2.07) \pm 0.12$; anteocular length $0.80(0.74-0.88) \pm 0.05$; length of antennal segments: I - 1.11 (1.04-1.21) \pm 0.08 ; II - $1.72(1.60-1.91) \pm 0.13$; III - 2.20 (1.84-2.42) \pm 0.23 ; IV - 3.84 (3.40-4.10) $\pm 0.38 ; \mathrm{V}$ - lacking; pronotal length 5.83 (5.18-6.35) \pm 0.52 , width $13.29(12.02-14.53) \pm 0.99$; scutellar length $8.72(8.35-9.12) \pm 0.33$, width 8.24 (7.52-8.88) \pm 0.50 ; corium length 12.08 (11.86-12.36) \pm 0.26 ; abdominal width 15.13 (14.03$16.03) \pm 0.88$; total length $20.61(19.04-22.38) \pm 1.53$.

Material examined. GUYANA, Essequibo: Kaieteur Fall (high forest), $\subsetneq$, 3.IX.1937, Richards \& Smart (BMNH 1937-776). BRAZIL, \& (NHMW); Pará: Mocajuba (Mangabeira), f, XI.1952, Orlando Rego (MNRJ); Bahia: 3, 2 f, 1859, Dr. Felder (NHMW); Espírito Santo: Conceição da Barra, \&, 27.V.1969, C.T. \& C. Elias (DZUP); São Paulo: Araras (Us. Santa Lúcia), f, 12.VII.1983, P.R. Cancian (UFRG); Campinas, f, XI.1986, L.O.T.M. (UFRG).

Distribution. Guyana (Essequibo), Brazil (Pará, Bahia, Espírito Santo, São Paulo).

Comments. Brachystethus vicinus is the sister group of B. improvisus, distinguishing from this species, in the males, by the shape of parameres, with acute apex, by the inconspicuous lobes of dorsal rim of pygophore and by the genital cup processes little visible dorsally. In the females, the posterior borders of gonocoxites 8 , projected in acute angle over the limit between laterotergites 8 and laterotergites 9 , also separate $B$. vicinus from $B$. improvisus. 

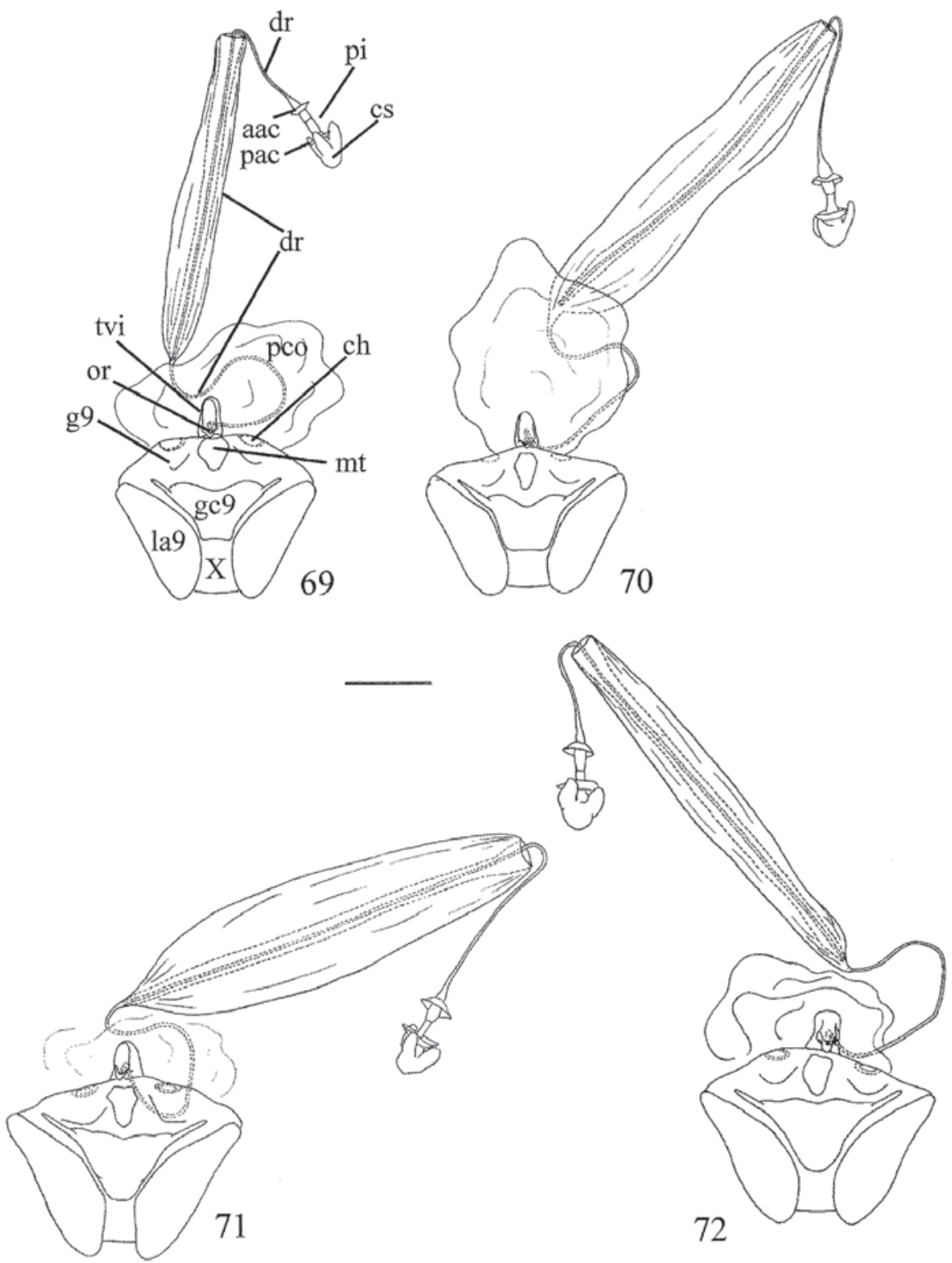

Figs. 69-72. Laterotergites, gonocoxites and gonapophyses of ninth segment, and ectodermical genital ducts, ventral: 69, Brachystethus vexillum; 70, B. geniculatus; 71, B. improvisus; 72 , B. vicinus (aac, anterior annular crest; ch, chitinellipsen; cs, capsula seminalis; dr, ductus receptaculi; g9, gonapophyses 9; gc9, gonocoxites 9; la9, laterotergites 9; mt, medial thickening of gonapophyses 9; or, orificium receptaculi; pac, posterior annular crest; pco, pars comunis; pi, pars intermedialis; tvi, thickening of vaginal intima; X, tenth segment). Scale, $1 \mathrm{~mm}$. 
Acknowledgments. To the curators of collections, for the loans. To Dr. Cláudio B. Carvalho (UFPR), Dr. Maria Helena Galileo (MCN), Dr. Luciane Marinoni (UFPR) and Dr. Rodney Cavichiolli (UFPR) for their critics and comments. To Dr. José Antônio M. Fernandes for the photographs, and to M.Sc. Cristiano F. Schwertner, for helping with the plates. To CNPq and CAPES for financial support.

\section{REFERENCES}

Амyot, C. J. B. \& Serville, J. G. A. 1843. Histoire Naturelle des Insectes. Hémiptères. Paris, Roret. $681 \mathrm{p}$.

Arnett, R. H ; Samuelson, G. A. et al. 1993. The insect and spider collections of the world. Gainesville, E.J. Briel. 309p.

Barcellos, A. \& Grazia, J. 2003. Cladistic analysis and biogeography of Brachystethus Laporte (Heteroptera, Pentatomidae, Edessinae). Zootaxa, Auckland, 256:1-14.

Berg, C. 1892. Nova Hemiptera faunarum Argentinae et Uruguayensis. An. Soc. Cient. argent., Buenos Aires, 33:5-11.

Bergroth, E. 1908. Enumeratio Pentatomidarum post catalogum Bruxellensem descriptarum. Mém. Soc. r. ent. Belg., Bruxelles, 15:131-200.

Bolívar, I. 1879. Hemípteros nuevos del Museo de Madrid. An. Soc. esp. Hist. nat., Madrid, 8(1): $133-146$.

Brailovsky, H. 1988. Hemiptera-Heteroptera de Mexico XXXVIII. Los Pentatomini de la Estación de Biología Tropical "Los Tuxtlas", Veracruz (Pentatomidae). An. Inst. Biol. Univ. Nac. Auton. Mex., Sér. Zool., Mexico, 58(1):69-154.

Brailovsky, H. \& Barrera, E. 1982. Hemiptera-Heteroptera de Mexico XXII. Nuevos registros de la tribu Pentatomini y descripción de una nueva especie del genero Pseudevoplitus Ruckes (Pentatomidae). An. Inst. Biol. Univ. Nac. Auton. Mex., Sér. Zool., Mexico, 52(1):231-246.

Brailovsky, H.; Cervantes, L. \& Mayorga, C. 1992. Hemiptera: Heteroptera de Mexico XLIV. Biología, estadios ninfales y fenologia de la tribu Pentatomini (Pentatomidae) en la Estación de Biología Tropical "Los Tuxtlas", Veracruz. Univ. Nac. Auton. Mex., Publ. esp. Inst. Biol., Mexico, 8:1-204.

Breddin, G. 1903. Beiträge zur Hemiptern-Fauna der Anden. Sber. Ges. naturf. Freunde Berl., Berlin, 8:366-383.

1904. Neue Rhynchotenausbeute aus Süd-Amerika. Societas ent., Berlin, 18(23):177-178.

1905. New Rhynchoten (Vorläufige Diagnosen). Societas ent., Berlin, 20(4):26-27.

Buckup, L. 1961. Os pentatomídeos do Rio Grande do Sul (Brasil). Hemiptera-HeteropteraPentatomidae. Iheringia, Sér. Zool., Porto Alegre, (16):1-24.

Burmeister, H. C. C. 1835. Handbuch der Entomologie. Berlin, G. Reimer, v. 2. 1050p.

Dallas, W. S. 1851. List of the specimens of Hemipterous insects of the British Museum I. London, British Museum. 390p., 15 pls.

Distant, W. L. 1880. Insecta, Rhynchota: Hemiptera-Heteroptera. In: Godman, F. D. \& Salvin, O. eds. Biologia Centrali-Americana. London, British Museum. v. 1, 462p.

DupuIs, C. 1955. Les genitalia des Hémiptères-Hétéroptères (genitalia externes des deux sexes, voies ectodermiques femeles). Mém. Mus. natn. Hist. nat. Paris, Sér. A, Zool., Paris, 6(4):183-278.

1970. Heteroptera. In: Tuxen, S. L. ed. Taxonomist's glossary of genitalia of insects. Copenhagen, Munksgaard. p. 190-208.

Fabricius, J. C. 1781. Classis VII: Rynchota. In: Species insectorum exhibentes eorum differentias specificas, synonyma auctorum, loca natalia, metamorphosin adjetis observationibus, descriptionibus. Hamburg, C. Ernst. v. 2, p. 313-397.

1787. Classis VII: Ryngota. In: Mantissa insectorum sistens eorum species nuper detectas: Adjectis characteribus genericis, differentiis specificis, emendationibus, observationibus. Hafniae, Impensis Christ. Gottl. Proft. v. 2, p. 1-382.

1794. Entomologia systematica emendata et aucta, secundum classes, ordines, genera, species adjectis, synonymis, locis, observationibus. Hafniae. v. 4, p. 66-127.

1803. Systema Rhyngotorum secundum ordines, genera, species adjectis, synonymis, locis, observationibus, descriptionibus. Brunsvigae. 335p. 
Froeschner, R. C. 1981. Heteroptera or true bugs of Ecuador: a partial catalog. Smithson. Contr. Zool.,Washington, 322:1-147.

Gmelin, J. F. 1793. Insecta Hemiptera. In: Caroli A. Linné Systema Naturae. Lipsie, ed. 13 aucta, reformata. tomo I, pars IV, p. 2041-2224.

GraZiA, J. 1977. Revisão dos pentatomíneos citados no "Quarto catálogo dos insetos que vivem nas plantas do Brasil" (Hemiptera-Pentatomidae-Pentatomini). Dusenia, Curitiba, 10(3):161-174. 1984. Pentatomini da Venezuela (Heteroptera, Pentatomidae). An. Soc. ent. Brasil, Porto Alegre, 13(1):71-81.

Grazia, J. \& Barcellos, A. 1991. Sobre o genus Paratibilis Ruckes (Heteroptera, Pentatomini). An. Soc. ent. Brasil, Londrina, 20(1):209-216.

GraZia-VIEIRA, J. \& CASINI, C. E. 1973. Lista preliminar dos heterópteros uruguaios da região Nordeste: Pentatomidae e Coreidae (Insecta; Heterotera). Iheringia, Sér. Zool., Porto Alegre, (44):55-63.

Herrich-SchäFfer, G. A. W. 1845. Die Wanzenartigen Insekten. Nürnberg, C.H. Zeh. v. 8, 48p. 1853. Die Wanzenartigen Insekten. Nurnberg, C.H. Zeh. v. 9, 348p.

Kirkaldy, G. W. 1909. Catalogue of the Hemiptera (Heteroptera). 1. Cimicidae. Berlin, Felix L. Dames. 392p.

Kormilev, N. A. 1951. Notas sobre hemipteros bolivianos con la descripción de un género y tres especies nuevos. Folia Univ. Cochabamba, Cochabamba, 5:35-45.

LAPORTE, F. L. DE. 1832. Essai d'une classification systematique de l'ordre des Hémiptères (HémiptèresHétéroptéres Latr.). Mag. Zool., London, 1(suppl.):1-88.

Lethierry, L. \& Severin, G. 1893. Catalogue général des Hemiptères; Pentatomidae 1. Bruxelles, F. Hayez. 286p.

Link, D. \& Grazia, J. 1987. Pentatomídeos da região central do Rio Grande do Sul. An. Soc. ent. Brasil, Porto Alegre, 16(1):115-129.

Maes, J. 1994. Catalogo de los Pentatomoidea (Heteroptera) de Nicaragua. Revta Nica. Ent., Managua, 28:1-29.

MAYr, G. L. 1866. Hemiptera in reise der Österreischischen Freggate Novara um die erde in den Jahren 1857, 1858, 1859. Zoologischer Teil, Wien. Bd. 2, abt. 1. 204p.

Pennington, M. S. 1920. Lista de los hemipteros heteropteros de la Republica Argentina. Parte I. Pentatomoidea, Coreoidea. Buenos Aires, 47p.

Pirán, A. P. 1955. Dos notas de sinonimia sobre Hemiptera neotropicales. Beitr. Ent., Berlin, 5(3/ 4):337-338.

_. 1956. Hemipteros raros o poco conocidos y no mencionados para las faunas de Brazil, Uruguay, Argentina, Paraguay y Bolivia. Revta Soc. urug. Ent., Montevideo, 1(1):29-35.

Rider, D. A. 1991. Rhyssocephala, new genus, with the description of three new species (Pentatomidae). JI N. Y. ent. Soc., New York, 99(4):583-610.

Rolston, L. H.; McDonald, F. J. D. \& Thomas, D. B., JR. 1980. A conspectus of Pentatomini genera of the Western Hemisphere; Part 1 (Hemiptera:Pentatomidae). Jl N. Y. ent. Soc., New York, $\mathbf{8 8}(2): 120-132$.

Rufinelli, A. \& Pirán, A. A. 1959. Hemipteros heterópteros del Uruguay. Boln Fac. Agrn. Mont., Montevideo, (51):12.

Signoret, V. 1851. Description de nouvelles espèces d'Hémiptères. Annls Soc. ent. Fr., Paris, 9 (2):329-348.

Silva, A. G.; Gonçalves, C. R. et al. 1968. Quarto catálogo dos insetos que vivem nas plantas do Brasil, seus parasitas e predadores. Rio de Janeiro, Ministério da Agricultura. t. I, parte II, $662 \mathrm{p}$.

Spinola, M. 1837. Essai sur les genres d'insectes appartenants à l'ordre des Hémiptères, Lin. ou Rhyngotes, Fab. et a la section des Hétéroptères, Dufour. Gênes, Yves Gravier. $383 p$.

STÅL, C. 1860. Bidrag till Rio Janeiro - traktens Hemipter-fauna. K. svenska VetenskAkad. Handl., Stockholm, 10(4):1-159.

1862. Hemiptera Mexicana. Enumeravit speciesque news descripsit. Stettin. ent. Ztg, Stettin, 23:81-118.

1867. Bidrag till hemipterernas systematik. Öfvers. K. VetenskAkad. Förh., Stockholm, 24(7):491-560.

1868. Hemiptera Fabriciana 1. K. svenska VetenskAkad. Handl., Stockholm, 7(11):1-148. 
1872. Enumeratio Hemipterorum 2. K. svenska VetenskAkad. Handl., Stockholm, 10(4):1-159.

1868. Catalogue of the specimens of Hemiptera-Heteroptera in the collection of the British Museum. London, British Museum. Part 3, p.419-599.

Zimsen, E. 1964. The type material of I. C. Fabricius. Copenhagen, Munksgaard. 656p.

Recebido em 04.11.2002; aceito em 04.08.2003.

Iheringia, Sér. Zool., Porto Alegre, 93(4):413-446, 30 de dezembro 2003 